\title{
Well-posedness of axisymmetric nonlinear surface waves on a ferrofluid jet
}

\author{
Zhan Wang $^{\mathrm{a}, \mathrm{b}, *}$, Jiaqi Yang ${ }^{\mathrm{a}}$ \\ ${ }^{a}$ Key Laboratory for Mechanics in Fluid Solid Coupling Systems, Institute of Mechanics, Chinese Academy of Sciences, \\ Beijing 100190, China \\ b School of Engineering Science, University of Chinese Academy of Sciences, Beijing 100049, China
}

Received 19 November 2018; revised 9 May 2019; accepted 20 May 2019

\begin{abstract}
We consider free surface waves propagating on a ferrofluid jet under a radial magnetic field. The waves investigated are axisymmetric solutions of the Euler equations, formulated in cylindrical coordinates, for an incompressible and inviscid ferrofluid flowing irrotationally, which satisfy the generalized Young-Laplace equation with magnetic stresses included and the kinematic condition on the free surface. The main objective of the present study is to solve a basic question on the theoretical side, i.e. the local well-posedness issue. We establish local existence and uniqueness of solutions for the initial value problem in Sobolev spaces, which are achieved based on analyses of the radially symmetric Dirichlet-Neumann operator and energy method.

(c) 2019 Published by Elsevier Inc.
\end{abstract}

MSC: 35Q35; 76B15

Keywords: Ferrofluids; Local well-posedness; Axisymmetric

\section{Introduction}

A ferrofluid is a colloidal liquid made of ferromagnetic nano-particles suspended in a carrier fluid and coated with surfactant to stop them from aggregating in the solution. In the presence

\footnotetext{
* Corresponding author.

E-mail addresses: zwang@imech.ac.cn (Z. Wang), yjq@imech.ac.cn (J. Yang).
} 
of an external magnetic field, random ferrofluid particles experience attractive magnetic forces and become highly magnetized. Ferrofluids receive growing attention due to their significant applications in various areas, such as industry [21] and medicine [23]. In addition, ferrofluids have been employed to study intriguing phenomena and fundamental aspects of fluid mechanics, e.g. to obtain insight into colloidal forces and their role in the stabilization of colloidal suspensions, which has led to new applications for ferrofluid-based emulsions [20].

In the current paper, we are interested in the following set-up: a ferrofluid jet is exposed to an azimuthal magnetic field generated by a current-carrying rod with the center along the jet axis. The induced axisymmetric body force has a stabilizing effect and allows disturbances to develop on the jet surface [5,6]. Rannacher \& Engel [22] confirmed via a linear stability analysis that the jet can indeed be stabilized and derived the cylindrical Kortwege-de Vries equation describing axisymmetric weakly nonlinear disturbances in the long-wave limit. They identified soliton solutions to this model and showed that solitons are of elevation waves (with a central hump) if $1<B<\frac{3}{2}$ and feature a depression profile (with a central dip) for $\frac{3}{2}<B<9$, where $B$ denotes the magnetic Bond number. Recently, Bourdin et al. [9] reported the first experimental observation of such axisymmetric waves and found a good agreement with the KdV predictions. Blyth and Părău [8] subsequently revisited this problem by solving the fully nonlinear equations numerically. Guyenne \& Părău [14] presented a new numerical method to simulate the time evolution of axisymmetric nonlinear waves on the surface of a ferrofluid jet. It is also worth mentioning that for $B<1$ the jet is unstable [4].

Although many numerical and experimental results have been obtained, as far as we know, there is no result on the well-posedness of axisymmetric ferrofluid jet, which is fundamental but has not been investigated. This paper is devoted to establish a local well-posedness result of the Cauchy problem for full Euler equations without vorticity. We first recall some well established results for fully nonlinear water-wave equations (without magnetic field). The small data problem was first addressed by Nalimov [19], but the first breakthrough in dealing with the local wellposedness issue with general data is due to $\mathrm{Wu}[25,26]$. If vorticity is considered, Christodoulou \& Lindblad [10] and Lindblad [18] established local existence and uniqueness results. Beyer \& Gunther [7] took into account the effects of surface tension and Lannes [15] treated the case of non-trivial bottom topography. The interested reader is referred to Ambrose \& Masmoudi [1,2], Coutand \& Shkoller [11], Shatah \& Zeng [24] and references therein for more results.

\subsection{Mathematical formulation}

We consider the inviscid, incompressible and irrotational flow of a liquid jet attached to the surface of a cylindrical metal rod of radius $b>0$. The liquid is of unit density and flows in the $z$-direction of a cylindrical coordinate system $(r, \theta, z)$. Additionally, we assume axisymmetric condition so that all variables are independent of $\theta$. The fluid occupying the region $S_{1}=\{b<$ $r<R+\eta(z, t)\}$ is subject to a static magnetic field, and the surrounding domain $S_{2}=\{r>$ $R+\eta(z, t)\}$ is a vacuum. Following [13], we denote by $\mathbf{H}_{1}, \mathbf{B}_{1}$ and $\mathbf{H}_{2}, \mathbf{B}_{2}$ the magnetic and induction fields in the fluid and in the vacuum respectively, and the relationships between them can be expressed as

$$
\mathbf{B}_{1}=\mu_{0}\left(\mathbf{H}_{1}+\mathbf{M}_{1}\left(\mathbf{H}_{1}\right)\right), \quad \mathbf{B}_{2}=\mu_{0} \mathbf{H}_{2},
$$

where $\mu_{0}$ is the magnetic permeability of free space and $\mathbf{M}_{1}$ is the prescribed magnetic intensity of the ferrofluid. Furthermore, we assume 


$$
\mathbf{M}_{1}\left(\mathbf{H}_{1}\right)=m_{1}\left(\left|\mathbf{H}_{1}\right|\right) \frac{\mathbf{H}_{1}}{\left|\mathbf{H}_{1}\right|}
$$

where $m_{1}$ is a prescribed nonnegative function, so that in particular $\mathbf{M}_{1}$ and $\mathbf{H}_{1}$ are collinear. According to Maxwell's equations the magnetic and induction fields are respectively irrotational and solenoidal. Therefore we can introduce magnetic potential functions $\psi_{1}$, $\psi_{2}$ with $\mathbf{H}_{1}=-\nabla_{x, y, z} \psi_{1}, \mathbf{H}_{2}=-\nabla_{x, y, z} \psi_{2}$, and then

$$
\begin{cases}\nabla_{x, y, z} \cdot\left(\mu\left(\left|\nabla_{x, y, z} \psi_{1}\right|\right) \nabla_{x, y, z} \psi_{1}\right)=0, & \text { in } S_{1}, \\ \Delta_{x, y, z} \psi_{2}=0, & \text { in } S_{2},\end{cases}
$$

where

$$
\mu(s)=1+\frac{m_{1}(s)}{s}
$$

is the magnetic permeability of the ferrofluid relative to that of free space. Ferrofluid flow is supposed to be irrotational, so that the velocity field $\mathbf{v}$ can be written as the gradient of a scalar velocity potential $\Phi$. The Euler equations for the ferrofluid are

$$
\mathbf{v}_{t}+\left(\mathbf{v} \cdot \nabla_{x, y, z}\right) \mathbf{v}=-\nabla_{x, y, z} p+\mu_{0}\left(\mathbf{M}_{1} \cdot \nabla_{x, y, z}\right) \mathbf{H}_{1},
$$

where $p$ is the pressure, and therefore the irrotationality gives

$$
\Phi_{t}+\frac{1}{2}\left|\nabla_{x, y, z} \Phi\right|^{2}-\mu_{0} \int_{0}^{\left|\mathbf{H}_{1}\right|} m_{1}(t) d t+p=c_{0},
$$

where $c_{0}$ is a constant. The magnetic boundary conditions are

$$
\mathbf{H}_{1} \cdot \mathbf{t}=\mathbf{H}_{2} \cdot \mathbf{t}, \quad \mathbf{B}_{1} \cdot \mathbf{n}=\mathbf{B}_{2} \cdot \mathbf{n},
$$

where $\mathbf{t}$ and $\mathbf{n}$ denote tangent and normal vectors to the free surface respectively, which can be rewritten as

$$
\psi_{2}-\left.\psi_{1}\right|_{r=R+\eta(z, t)}=0, \quad \psi_{2 n}-\left.\mu\left(\left|\nabla_{x, y, z} \psi_{1}\right|\right) \psi_{1 n}\right|_{r=R+\eta(, z, t)}=0 .
$$

The generalized Young-Laplace equation gives the dynamic boundary condition

$$
p+\frac{\mu_{0}}{2}\left(\mathbf{M}_{1} \cdot \mathbf{n}\right)^{2}=\gamma \kappa
$$

where $\gamma>0$ is the coefficient of surface tension and $\kappa$ is the mean curvature of the free surface. It follows from (1) that

$$
\Phi_{t}+\frac{1}{2}\left|\nabla_{x, y, z} \Phi\right|^{2}-\mu_{0} v\left(\left|\nabla_{x, y, z} \psi_{1}\right|\right)+\gamma \kappa-\left.\frac{\mu_{0}}{2}\left(\mu\left(\left|\nabla_{x, y, z} \psi_{1}\right|\right)-1\right)^{2}\left(\psi_{1 n}\right)^{2}\right|_{r=R+\eta(z, t)}=c_{0}
$$


with

$$
v(s)=\int_{0}^{s} m_{1}(t) d t
$$

Finally, the kinematic boundary condition reads

$$
\left(\partial_{t}+\mathbf{v} \cdot \nabla_{x, y, z}\right)(r-R-\eta(z, t))=0,
$$

that is

$$
-\eta_{t}+\Phi_{r}-\left.\Phi_{z} \eta_{z}\right|_{r=R+\eta(z, t)}=0
$$

The conditions at $r=b$ are impermeability conditions for both fluid and magnetic field

$$
\Phi_{r}=\psi_{1 r}=0 .
$$

The constant $c_{0}$ is selected so that

$$
\mathbf{H}_{1}=\frac{J}{2 \pi r} \mathbf{e}_{\theta}, \quad \mathbf{H}_{2}=\frac{J}{2 \pi r} \mathbf{e}_{\theta}, \quad \mathbf{v}=0, \quad \eta=0
$$

is a solution to the above equations; we therefore set $c_{0}=-\mu_{0} v\left(\frac{J}{2 \pi R}\right)+\frac{\gamma}{R}$. Upon noticing that $\eta$ and $\Phi$ are independent of $\theta$ for axisymmetric waves, one finds that $\psi_{1}=\psi_{2}=-\frac{J \theta}{2 \pi}$, hence one can decouple the hydrodynamic problem from the magnetic problem, see [13]. Finally, it boils down to solve

$$
\begin{cases}\Phi_{r r}+\frac{1}{r} \Phi_{r}+\Phi_{z z}=0, & \text { in } b<r<R+\eta(z, t) \\ \Phi_{r}=0, & \text { on } r=b\end{cases}
$$

and

$$
\begin{cases}-\eta_{t}+\Phi_{r}-\Phi_{z} \eta_{z}=0, & \text { on } r=R+\eta(z, t) \\ \partial_{t} \Phi+\frac{1}{2}\left(\Phi_{r}^{2}+\Phi_{z}^{2}\right)-\mu_{0}\left(v\left(\frac{J}{2 \pi(R+\eta)}\right)-v\left(\frac{J}{2 \pi}\right)\right)+\gamma \kappa=0, & \text { on } r=R+\eta(z, t)\end{cases}
$$

where

$$
\kappa=-\frac{\eta_{z z}}{\left(R+\eta_{z}^{2}\right)^{\frac{3}{2}}}+\left(\frac{1}{(R+\eta)\left(R+\eta_{z}^{2}\right)^{\frac{1}{2}}}-\frac{1}{R}\right):=\kappa_{1}+\kappa_{2}
$$

and

$$
v(s)=\int_{0}^{s} m_{1}(t) d t
$$


In order to prove the local well-posedness of system (2)-(3), it is useful to reduce the above system to the following Zakharov-Craig-Sulem formulation

$$
\left\{\begin{array}{l}
\eta_{t}-\mathcal{G}[\eta] \eta=0 \\
\partial_{t} \psi+\frac{1}{2} \psi_{z}^{2}-\frac{\left(\mathcal{G}[\eta] \psi+\eta_{z} \psi_{z}\right)^{2}}{2\left(1+\eta_{z}^{2}\right)}-\mu_{0} v\left(\frac{J}{2 \pi(R+\eta)}\right)+\mu_{0} v\left(\frac{J}{2 \pi}\right)+\gamma\left(\kappa_{1}+\kappa_{2}\right)=0
\end{array}\right.
$$

where $\psi(z, t)=: \Phi(z, R+\eta(z, t), t)$ and $\mathcal{G}[\eta]$ is the Dirichlet-Neumann operator (see (16) for details).

\subsection{Main result}

Theorem 1. Choose $t_{0} \geqslant 1$ and $N \geqslant 5$. Let $U^{0}$ satisfy (8) and $\mathcal{E}^{N}\left(U^{0}\right)<\infty$. There exist $T>0$ and a unique solution $U \in E_{T}^{N}$ to system (4) with initial data $U^{0}$, where $\mathcal{E}^{N}\left(U^{0}\right)$ and $E_{T}^{N}$ are defined by (21) and (30) respectively.

Remark 1. Although $\gamma$ is supposed to be positive in this paper, we can still obtain the local well-posedness for $\gamma=0$ if we assume that $\mathfrak{a}\left(U^{0}\right)>0$ with $\mathfrak{a}\left(U^{0}\right)$ defined in (23). It can be achieved by a similar argument due to $\frac{J}{2 \pi\left(1+\eta_{0}\right)^{2}} m_{1}\left(\frac{J}{2 \pi\left(1+\eta_{0}\right)}\right)>0$.

Remark 2. Using the estimates obtained in the current paper and the argument of Chapter 4 in [16], one can also obtain the following stability result without any essentially difficulties.

If $U^{\prime}$ solves the system (4) with initial data $U^{\prime 0}$, then $U-U^{\prime}$ satisfies for all $0 \leqslant t \leqslant T$,

$$
\mathcal{E}^{N}\left(\left(U-U^{\prime}\right)(t)\right) \leqslant C\left(c_{U}, c_{U^{\prime}}\right) \mathcal{E}^{N}\left(U^{0}-U^{\prime 0}\right),
$$

where $c_{U}=\sup _{0 \leqslant t \leqslant T} \mathcal{E}^{N}(U(t))$ and $c_{U^{\prime}}=\sup _{0 \leqslant t \leqslant T} \mathcal{E}^{N}\left(U^{\prime}(t)\right)$.

\subsection{Comments}

We will adopt the framework of Lannes (see [15-17]) to prove the local existence and uniqueness of solutions to the Cauchy problem of system (4). Consider the classical 2-D gravity water-wave equations in domain $\left\{(z, r) \in \mathbb{R}^{2}: b<r<R+\eta(z, t)\right\}$, following Zakharov [27] or Craig \& Sulem [12], the system can be written as

$$
\left\{\begin{array}{l}
\eta_{t}=\mathcal{G}[\eta] \psi, \\
\psi_{t}+g \eta+\frac{1}{2} \psi_{z}^{2}-\frac{\left(\mathcal{G}[\eta] \psi+\eta_{z} \psi_{z}\right)^{2}}{2\left(1+\eta_{z}^{2}\right)}=\gamma \partial_{z}\left(\frac{\eta_{z}}{\sqrt{1+\eta_{z}^{2}}}\right),
\end{array}\right.
$$

where $g$ is the acceleration due to gravity, $\mathcal{G}[\eta] \psi:=\left.\sqrt{1+\eta_{z}^{2}}\left(\Phi_{r}-\eta_{z} \Phi_{z}\right)\right|_{r=R+\eta}$, and the velocity potential $\Phi$ satisfies

$$
\begin{cases}\Phi_{r r}+\Phi_{z z}=0, & \text { in } b<r<R+\eta(z, t), \\ \left.\Phi\right|_{r=R+\eta}=\psi, & \left.\Phi_{r}\right|_{r=b}=0 .\end{cases}
$$


To obtain the local well-posedness of system (5), it is necessary to reduce it to a quasilinear form. It is well known that the linearization of (5) around $\eta=0$ and $\psi=0$ is

$$
\left\{\begin{array}{l}
\eta_{t}-\mathcal{G}[0] \psi=0 \\
\psi_{t}+\left(g-\gamma \partial_{z z}\right) \eta=0
\end{array}\right.
$$

However, to deal with the fully nonlinear problem, we must linearize the system around a nontrivial state, thus the situation becomes much more complicated. Lannes' key observation is that system (5) can be rewritten in a similar form by introducing 'good' unknowns $\eta_{(\alpha)}=\partial^{\alpha} \eta$ and $\psi_{(\alpha)}=\partial^{\alpha} \psi-\underline{Z} \partial^{\alpha} \eta$ where $\alpha=\left(\alpha_{0}, \alpha_{1}\right)^{\top}$ is a multiple index. It follows that

$$
\left\{\begin{array}{l}
\partial_{t} \eta_{(\alpha)}+\partial_{z}\left(\underline{V} \eta_{(\alpha)}\right)-\mathcal{G}[\eta] \psi_{(\alpha)}+\text { subprincipal terms }=\text { lower order terms } \\
\partial_{t} \psi_{(\alpha)}+\underline{V} \cdot \partial_{z} \psi_{(\alpha)}-\gamma \partial_{z} \mathcal{K}\left[\eta_{z}\right] \partial_{z} \eta_{(\alpha)}+\text { subprincipal terms }=\text { lower order terms }
\end{array}\right.
$$

where

$$
\underline{Z}=\frac{\mathcal{G}[\eta] \psi+\eta_{z} \psi_{z}}{1+\eta_{z}^{2}}, \quad \underline{V}=\psi_{z}-\underline{Z} \eta_{z}
$$

are the vertical and horizontal velocities at the surface respectively, and

$$
\mathcal{K}\left[\eta_{z}\right]=\frac{1}{\left(1+\eta_{z}^{2}\right)^{\frac{3}{2}}}
$$

The remaining part is standard since the quasilinear system (7) can be handled by symmetrization and energy estimates. For our problem, the velocity potential $\Phi$ satisfies (2) rather than (6), so we should establish the properties of the Dirichlet-Neumann operator in the radial symmetric case. On the other hand, $\mathcal{G}[\eta]$ is no longer a self-adjoint operator in this case, therefore a new self-adjoint operator needs to be introduced, and subsequently, the linearization formulas and symmetrizers also need to be modified.

The paper is organized as follows. In Section 2, we establish the properties of the DirichletNeumann operator in the axisymmetric case. In Section 3 and Section 4, we focus on the linearization formulas and subsequently derive a quasilinear system for high-order derivatives of unknowns. Finally, we complete the proof of Theorem 1 in Section 5.

Since our interest is the local well-posedness of the Cauchy problem, without loss of generality, we assume $\mu_{0}=\gamma=R=1$ in the rest of the paper.

\section{Preliminary results}

In this section, we explore the Dirichlet-Neumann operator in the axisymmetric situation via investigating a boundary value problem for a linear elliptic PDE as [3,15]. It is noted that, in contrast to [3,15], the elliptic operator $\partial_{r}\left(r \partial_{r} \bullet\right)+\partial_{z}\left(r \partial_{z} \bullet\right)$ is of variable coefficients rather than constant coefficients. Throughout this section, we assume that the following assumption is always satisfied

$$
\exists h_{0}>0, \quad \inf _{z \in \mathbb{R}}(1-b+\eta(z)) \geqslant h_{0} .
$$




\subsection{Elliptic estimates on strip}

Since the velocity potential satisfies (2), it is natural to study the following boundary value problem

$$
\left\{\begin{array}{l}
\partial_{r}\left(r \partial_{r} \Phi\right)+\partial_{z}\left(r \partial_{z} \Phi\right)=0, \quad b<r<1+\eta \\
\left.\Phi\right|_{r=1+\eta}=\psi,\left.\quad\left(r \partial_{r} \Phi\right)\right|_{r=b}=0
\end{array}\right.
$$

We introduce the flat strip $\mathcal{S}=\mathbb{R} \times(0,1)$, and due to the assumption (8), one can define a diffeomorphism $S$ mapping $\mathcal{S}$ onto the fluid domain $\Omega$ as follows

$$
\begin{aligned}
\mathcal{S} & \rightarrow \Omega \\
(z, r) & \mapsto(z, r+\sigma(z, r)),
\end{aligned}
$$

with

$$
\sigma=(\eta(z)-b) r+b .
$$

Following the arguments of Lemma 2.5 of [15], with a slight modification, one can easily obtain that problem (9) is equivalent to the following boundary value problem

$$
\begin{cases}\nabla \cdot P[\eta] \nabla \phi=0, & \text { in } \mathcal{S}, \\ \left.\phi\right|_{r=1}=\psi, & -\left.(0,1) \cdot P[\eta] \nabla \phi\right|_{r=0}=0\end{cases}
$$

with $\phi=\Phi \circ S$ and the matrix $P[\eta]$ given by

$$
P[\eta]:=(r+\sigma)\left(\begin{array}{cc}
1+\partial_{r} \sigma & -\partial_{z} \sigma \\
-\partial_{z} \sigma & \frac{1+\left(\partial_{z} \sigma\right)^{2}}{1+\partial_{r} \sigma}
\end{array}\right)
$$

We can also write $P[\eta]$ as $[r+b(1-r)]+Q[\eta]$, where

$$
Q[\eta]:=\left(\begin{array}{cc}
\partial_{r} \sigma & -\partial_{z} \sigma \\
-\partial_{z} \sigma & \frac{-\partial_{r} \sigma+\left(\partial_{z} \sigma\right)^{2}}{1+\partial_{r} \sigma}
\end{array}\right)+r \eta\left(\begin{array}{cc}
1+\partial_{r} \sigma & -\partial_{z} \sigma \\
-\partial_{z} \sigma & \frac{1+\left(\partial_{z} \sigma\right)^{2}}{1+\partial_{r} \sigma}
\end{array}\right) .
$$

In the subsequent analyses, we use the condensed notation

$$
A_{s}=B_{s}+\left\langle C_{s}\right\rangle_{s>\underline{s}}
$$

to say that $A_{s}=B_{s}$ if $s \leqslant \underline{s}$ and $A_{s}=B_{s}+C_{s}$ if $s>\underline{s}$. For all $1 \leqslant p \leqslant \infty,|\cdot|_{p}$ denotes the classical norm of $L^{p}(\Omega)$ while $\|\cdot\|_{p}$ stands for the canonical norm of $L^{p}(\mathcal{S})$. For all $s \in \mathbb{R}$, $H^{s}(\mathbb{R})$ is the classical Sobolev space defined as

$$
H^{s}(\mathbb{R})=\left\{u \in \mathcal{S}^{\prime},|u|_{H^{s}}:=\left|\left(1-\partial_{z z}\right)^{\frac{s}{2}} u\right|_{2}<\infty\right\}
$$

We also define the Sobolev space 


$$
\dot{H}^{s+\frac{1}{2}}(\mathbb{R})=\left\{u \in L_{\text {loc }}^{2}(\mathbb{R}), \partial_{z} u \in H^{s-\frac{1}{2}}(\mathbb{R})\right\}
$$

endowed with the norm $|u|_{\dot{H}^{s+\frac{1}{2}}}=\left|\partial_{z} u\right|_{H^{s-\frac{1}{2}}} \cdot\|\cdot\|_{L^{\infty} H^{s}}$ denotes the norm of $L^{\infty}\left([0,1] ; H^{s}(\mathbb{R})\right)$ and $\|\cdot\|_{\infty, T}$ stands for the norm of $L^{\infty}\left([0, T] ; L^{\infty}(\mathbb{R})\right)$. Denoting $\nabla=\left(\partial_{z}, \partial_{r}\right)^{\top}$, we next state some properties of $Q[\eta]$.

Proposition 2. Let $t_{0}>\frac{1}{2}, s \geqslant 0$, and $\eta \in H^{t_{0}+1} \cap H^{s+1}(\mathbb{R})$ be such that (8) is satisfied.

(1) One has

$$
\|Q[\eta]\|_{L^{\infty} H^{s}} \leqslant C\left(h_{0}^{-1},\|\eta\|_{L^{\infty} H^{t_{0}}}+\|\nabla \eta\|_{L^{\infty} H^{t_{0}}}\right)\left(\|\eta\|_{L^{\infty} H^{s}}+\|\nabla \eta\|_{L^{\infty} H^{s}}\right)
$$

and when $\eta$ is also time dependent,

$$
\left\|\partial_{t} Q[\eta]\right\|_{\infty, T} \leqslant C\left(h_{0}^{-1},\|\eta\|_{\infty, T}+\|\nabla \eta\|_{\infty, T}\right)\left(\left\|\partial_{t} \eta\right\|_{\infty, T}+\left\|\nabla \partial_{t} \eta\right\|_{\infty, T}\right) .
$$

(2) For all $j \geqslant 1$ and $\mathbf{h} \in H^{t_{0}+1} \cap H^{s+1}(\mathbb{R})^{j}$, if we denote by $Q^{(j)}[\eta](\mathbf{h})$ the $j$-th derivative of $\eta \mapsto Q[\eta]$ in the direction $\mathbf{h}$, then

$$
\begin{aligned}
& \left\|Q^{(j)}[\eta](\mathbf{h})\right\|_{L^{\infty} H^{s}} \\
\leqslant & C\left(h_{0}^{-1},\|\eta\|_{L^{\infty} H^{t_{0}}}+\|\nabla \eta\|_{L^{\infty} H^{t_{0}}}\right) \\
\times & \left(\sum_{k=1}^{j}\left|h_{k}\right|_{H^{s+1}} \prod_{l \neq k}\left|h_{l}\right|_{H^{t_{0}+1}}+\left\langle\left(\|\eta\|_{L^{\infty} H^{s}}+\|\nabla \eta\|_{L^{\infty} H^{s}}\right) \prod_{k=1}^{j}\left|h_{k}\right|_{H^{t_{0}+1}}\right\rangle_{s>t_{0}}\right) .
\end{aligned}
$$

(3) The matrix $[r+b(1-r)]+Q[\eta]$ is coercive in the sense that

$$
\forall \Theta \in \mathbb{R}^{2}, \quad|\Theta|^{2} \leqslant C k[\eta]([b(1-r)+r]+Q[\eta]) \Theta \cdot \Theta,
$$

with

$$
k[\eta]:=\frac{1}{b}\left(1+\|\sigma\|_{\infty}\right)+\frac{1}{b h_{0}}\left(1+\left\|\partial_{z} \sigma\right\|_{\infty}\right) .
$$

Proof. (1) and (2) can be obtained from the following tame product and Moser's estimate (see Proposition 2.1 in [3]),

$$
|f g|_{H^{s}} \leqslant C\left(|f|_{H^{t_{0}}}|g|_{H^{s}}+\left\langle|f|_{H^{s}}|g|_{H^{t_{0}}}\right\rangle_{s>t_{0}}\right), \quad \text { for } t_{0}>\frac{1}{2} \text { and } s \geqslant 0
$$

and for all $F \in C^{\infty}\left(\mathbb{R}^{n}, \mathbb{R}^{m}\right)$ such that $F(0)=0$,

$$
|F(u)|_{H^{s}} \leqslant C\left(|u|_{\infty}\right)|u|_{H^{s}}, \quad s \geqslant 0 .
$$

It is not difficult to check that $([b(1-r)+r]+Q[\eta]) \Theta \cdot \Theta=\frac{r+\sigma}{1+\partial_{z} \sigma}|B \Theta|^{2}$, where 


$$
B=\left(\begin{array}{cc}
1+\partial_{r} \sigma & \partial_{z} \sigma \\
0 & 1
\end{array}\right)
$$

Upon noting that

$$
B^{-1}=\frac{1}{\partial_{z} \sigma}\left(\begin{array}{cc}
1 & \partial_{z} \sigma \\
0 & 1+\partial_{z} \sigma
\end{array}\right)
$$

and

$$
|\Theta| \leqslant|B \Theta|\left|B^{-1}\right|_{\mathbb{R}^{2} \mapsto \mathbb{R}^{2}},
$$

one can obtain (3).

Next, we consider the following boundary value problem

$$
\left\{\begin{array}{l}
\nabla \cdot([r+b(1-r)]+Q[\eta]) \nabla u=\nabla \cdot \mathbf{g}, \quad \text { in } \mathcal{S}, \\
\left.u\right|_{r=1}=0, \quad-\left.(0,1) \cdot P[\eta] \nabla u\right|_{r=0}=-\left.(0,1) \cdot \mathbf{g}\right|_{r=0} .
\end{array}\right.
$$

In the following, we set

$$
M[\eta]:=C\left(h_{0}^{-1},\|\eta\|_{L^{\infty} H^{t_{0}+1}}+\|\nabla \eta\|_{L^{\infty} H^{t_{0}+1}}\right),
$$

where $C(\cdot)$ is a non-decreasing function of its arguments.

Proposition 3. Let $t_{0}>\frac{1}{2}, s \geqslant 0$ and $\eta \in H^{t_{0}+2} \cap H^{s+1}(\mathbb{R})$ be such that (8) is satisfied. Then for all $\mathbf{g} \in C\left([0,1] ; H^{s}(\mathbb{R})\right)^{2}$, there exists a unique variational solution $u$ to (12) and satisfying the estimate

$$
\left\|\Lambda^{s} \nabla u\right\|_{2} \leqslant M[\eta]\left(\left\|\Lambda^{s} \mathbf{g}\right\|_{2}+\left\langle\left(\|\eta\|_{L^{\infty} H^{s}}+\|\nabla \eta\|_{L^{\infty} H^{s}}\right)\left\|\Lambda^{s} \mathbf{g}\right\|_{2}\right\rangle_{s>t_{0}+1}\right) .
$$

Proof. A standard argument shows the existence of solution. We next give detailed proof of the inequality, which is similar to the proof of Proposition 2.4 in [3] with some modifications. Let $\chi(\cdot)$ be a smooth and compactly supported function such that $\chi(\xi)=1$ in a neighborhood of $\xi=0$, and defined $\Lambda_{h}:=\Lambda * \chi\left(h\left|i \partial_{z}\right|\right)$. Letting $\Lambda_{h}^{2 s} u$ be a test function, one can get

$$
\int_{\mathcal{S}}([r+b(1-r)]+Q[\eta]) \nabla u \cdot \nabla \Lambda_{h}^{2 s} u=\int_{\mathcal{S}} \mathbf{g} \cdot \nabla \Lambda_{h}^{2 s} u
$$

which implies that

$$
\int_{\mathcal{S}} \Lambda_{h}^{s}([r+b(1-r)]+Q[\eta]) \nabla u \cdot \nabla v_{h}=\int_{\mathcal{S}} \mathbf{g} \cdot \nabla v_{h}
$$

with $v_{h}:=\Lambda_{h}^{s} u$ and thereby 


$$
\int_{\mathcal{S}}([r+b(1-r)]+Q[\eta]) \nabla v_{h} \cdot \nabla v_{h}=\int_{\mathcal{S}}\left(\mathbf{g} \cdot \nabla v_{h}-\left[\Lambda_{h}^{s}, Q[\eta]\right] \nabla u \cdot \nabla v_{h}\right) .
$$

From the third property of Proposition 2, one can obtain

$$
k[\eta]^{-1}\left\|\Lambda_{h}^{s} \nabla u\right\|_{2} \leqslant\left\|\left[\Lambda_{h}^{s}, Q[\eta]\right] \nabla u\right\|_{2}+\left\|\Lambda_{h}^{s} \mathbf{g}\right\|_{2} .
$$

Using the commutator estimates of Corollary 2.1 in [3], and letting $h \rightarrow 0$, one has

$$
\begin{aligned}
k[\eta]^{-1}\left\|\Lambda^{s} \nabla u\right\|_{2} \leqslant & C\left\|\Lambda^{s} \mathbf{g}\right\|_{2}+\|Q[\eta]\|_{L^{\infty} H^{t_{0}+1}}\left\|\Lambda^{s-1} \nabla u\right\|_{2} \\
& +C\left\langle\|Q[\eta]\|_{L^{\infty} H^{s}}\left\|\Lambda^{t_{0}} \nabla u\right\|_{2}\right\rangle_{s>t_{0}+1},
\end{aligned}
$$

and therefore

$$
\begin{aligned}
\left\|\Lambda^{s} \nabla u\right\|_{2} \leqslant & C\left(k[\eta],\|Q[\eta]\|_{L^{\infty} H^{t_{0}+1}}\right)\left(\left\|\Lambda^{s} \mathbf{g}\right\|_{2}+\left\|\Lambda^{s-1} \nabla u\right\|_{2}\right. \\
& \left.+\left\langle\|Q[\eta]\|_{L^{\infty} H^{s}}\left\|\Lambda^{t_{0}} \nabla u\right\|_{2}\right\rangle_{s>t_{0}+1}\right) .
\end{aligned}
$$

In particular,

$$
\|\nabla u\|_{2} \leqslant C k[\eta]\|\mathbf{g}\|_{2}, \quad\left\|\Lambda^{t_{0}} \nabla u\right\|_{2} \leqslant C\left(k[\eta],\|Q[\eta]\|_{L^{\infty} H^{t_{0}+1}}\right)\left\|\Lambda^{t_{0}} \mathbf{g}\right\|_{2} .
$$

One can finally obtain the estimate from (13), (14) and Proposition 2.

Next, we define $u^{b}$ as the solution to the boundary value problem

$$
\left\{\begin{array}{l}
\nabla \cdot([r+b(1-r)]+Q[\eta]) \nabla u^{b}=0, \quad \text { in } \mathcal{S} \\
\left.u^{b}\right|_{r=1}=u, \quad-\left.(0,1) \cdot P[\eta] \nabla u^{b}\right|_{r=0}=0
\end{array}\right.
$$

and also define $u^{\dagger}$ as

$$
\forall r \in[0,1], \quad u^{\dagger}(\cdot, r)=\chi\left((r-1)\left|i \partial_{z}\right|\right) u
$$

where $\chi$ is a smooth and compactly supported function such that $\chi(0)=1$.

Corollary 4. For $t_{0}>\frac{1}{2}$ and $s \geqslant 0$, let $\eta \in H^{t_{0}+2} \cap H^{s+1}(\mathbb{R})$ be such that (8) is satisfied. Then for all $u \in H^{s+\frac{1}{2}}(\mathbb{R})$, there exists a unique solution $u^{b}$, and

$$
\left\|\Lambda^{s} \nabla u^{\mathrm{b}}\right\|_{2} \leqslant M[\eta]\left(|\mathfrak{B} u|_{H^{s}}+\left\langle\left(\|\eta\|_{L^{\infty} H^{s}}+\|\nabla \eta\|_{L^{\infty} H^{s}}\right)|\mathfrak{B} u|_{H^{t_{0}}}\right\rangle_{s>t_{0}+1}\right)
$$

where $\mathfrak{B}:=\frac{\left|i \partial_{z}\right|}{1+\left|i \partial_{z}\right|^{\frac{1}{2}}}$. 
Proof. Seeking $u^{b}$ with the form $u^{b}=v+u^{\dagger}$ yields

$$
\left\{\begin{array}{l}
\nabla \cdot([r+b(1-r)]+Q[\eta]) \nabla v=-\nabla \cdot([r+b(1-r)]+Q[\eta]) \nabla u^{\dagger} \\
\left.v\right|_{r=1}=0, \quad-\left.(0,1) \cdot P[\eta] \nabla v\right|_{r=0}=\left.(0,1) \cdot P[\eta] \nabla u^{\dagger}\right|_{r=0} .
\end{array}\right.
$$

From Proposition 3, one has

$$
\left\|\Lambda^{s} \nabla v\right\|_{2} \leqslant M[\eta]\left(\left\|\Lambda^{s} \nabla u^{\dagger}\right\|_{2}+\left\langle\left(\|\eta\|_{L^{\infty} H^{s}}+\|\nabla \eta\|_{L^{\infty} H^{s}}\right)\left\|\Lambda^{t_{0}} \nabla u^{\dagger}\right\|_{2}\right\rangle_{s>t_{0}+1}\right) .
$$

Now, the corollary follows from the following fact (see Proposition 2.2 in [3])

$$
C_{1}|\mathfrak{B} u|_{H^{s}} \leqslant\left\|\Lambda^{s} \nabla u^{\dagger}\right\|_{2} \leqslant C_{2}|\mathfrak{B} u|_{H^{s}}
$$

\subsection{Dirichlet-Neumann operator}

The standard Dirichlet-Neumann operator is defined as

$$
\mathcal{G}[\eta] \psi:=\left.\sqrt{1+\eta_{z}^{2}}\left(\Phi_{r}-\eta_{z} \Phi_{z}\right)\right|_{r=1+\eta}
$$

Since $\mathcal{G}[\eta]$ is not self-adjoint in the axisymmetric situation, we introduce a new operator $\tilde{\mathcal{G}}[\eta]$ as

$$
\tilde{\mathcal{G}}[\eta]:=(1+\eta) \mathcal{G}[\eta] .
$$

We remark that the definition of the operator $\tilde{\mathcal{G}}[\eta]$ is natural. Actually, unlike the classical situation, the elliptic operator takes the form of $\partial_{r}\left(r \partial_{r} \bullet\right)+\partial_{z}\left(r \partial_{z} \bullet\right)$ in the radially symmetric case. It follows that the Neumann operator (corresponding to the Neumann boundary condition) on the free surface should be $\left.r \partial_{n}(\bullet)\right|_{(1+\eta)}$ instead of $\left.\partial_{n}(\bullet)\right|_{(1+\eta)}$. Furthermore, corresponding to system (10), $\tilde{\mathcal{G}}[\eta] \psi$ can be written as

$$
\tilde{\mathcal{G}}[\eta] \psi=\left.(0,1) \cdot P[\eta] \nabla \phi\right|_{r=1},
$$

where $\phi$ solves $(10)$, i.e. $\operatorname{div}(P[\eta] \phi)=0$. We next focus on the properties of the self-adjoint operator $\tilde{\mathcal{G}}[\eta]$.

Proposition 5. Let $t_{0}>\frac{1}{2}$ and $\eta \in H^{t_{0}+2}(\mathbb{R})$ be such that (8) is satisfied. Then

(1) $\tilde{\mathcal{G}}[\eta]$ is self-adjoint, namely,

$$
\forall u, v \in \dot{H}^{\frac{1}{2}}(\mathbb{R}), \quad(u, \tilde{\mathcal{G}}[\eta] v)=(v, \tilde{\mathcal{G}}[\eta] u) .
$$

(2) $\forall u, v \in \dot{H}^{\frac{1}{2}}(\mathbb{R})$, the following inequality holds

$$
|(u, \tilde{\mathcal{G}}[\eta] v)| \leqslant(u, \tilde{\mathcal{G}}[\eta] u)^{\frac{1}{2}}(v, \tilde{\mathcal{G}}[\eta] v)^{\frac{1}{2}} .
$$


Proof. The proposition can be obtained from the following identity

$$
\begin{aligned}
(u, \tilde{\mathcal{G}}[\eta] v) & =\int_{\mathcal{S}}([r+b(1-r)]+Q[\eta]) \nabla u^{b} \cdot \nabla v^{b} \\
& =\int_{\mathcal{S}}([r+b(1-r)]+Q[\eta])^{\frac{1}{2}} \nabla u^{b} \cdot([r+b(1-r)]+Q[\eta])^{\frac{1}{2}} \nabla v^{b},
\end{aligned}
$$

where $([r+b(1-r)]+Q[\eta])^{\frac{1}{2}}$ stands for the square root of the positive definite matrix $([r+$ $b(1-r)]+Q[\eta])^{\frac{1}{2}}$.

Proposition 6. Let $t_{0}>\frac{1}{2}$ and $\eta \in H^{t_{0}+2}$ be such that (8) is satisfied.

(1) For all $u \in \dot{H}^{s+\frac{1}{2}}(\mathbb{R}), 0 \leqslant s \leqslant t_{0}+1, j \in \mathbb{N}$ and $\mathbf{h} \in\left(H^{s \vee t_{0}+1}(\mathbb{R})\right)^{j}$, one has

$$
\left|d_{\eta}^{j} \tilde{\mathcal{G}}(\mathbf{h}) u\right|_{H^{s-\frac{1}{2}}} \leqslant M[\eta]|\mathfrak{B} u|_{H^{s}} \prod_{k=1}^{j}\left|h_{k}\right|_{H^{s \vee t_{0}+1}} .
$$

Hereafter, the notation $a \vee b$ stands for $\max \{a, b\}$.

(2) For all $u \in \dot{H}^{t_{0} \vee s+1}(\mathbb{R}), 0 \leqslant s \leqslant t_{0}+\frac{1}{2}, 1 \leqslant l \leqslant j$, and $\mathbf{h} \in\left(H^{t_{0}+2}(\mathbb{R})\right)^{j}$, one has

$$
\left|d_{\eta}^{j} \tilde{\mathcal{G}}(\mathbf{h}) u\right|_{H^{s-\frac{1}{2}}} \leqslant M[\eta]|\mathfrak{B} u|_{H^{s \vee t_{0}+\frac{1}{2}}}\left|h_{l}\right|_{H^{s+\frac{1}{2}}} \prod_{k \neq l}\left|h_{k}\right|_{H^{s \vee t_{0}+\frac{3}{2}}} \cdot
$$

Remark 3. One can also obtain the estimates for $s \geqslant t_{0}+1$ from the following proof and (2) of Proposition 2. In particular, when $j=0$, for any $s \geqslant 0$ one has

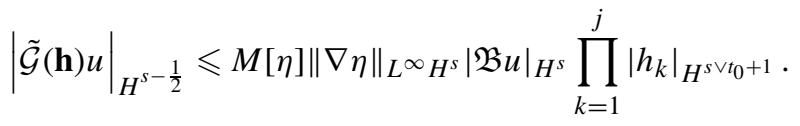

Proof. One can obtain (1) by using (2) of Proposition 2, Corollary 4, and slightly modifying the argument of Proposition 3.3 in [3]. (2) can be obtained by following a similar argument given in B.2.2 in [17], and using the estimate (see Lemma 14 of [17])

$$
\left\|\Lambda^{s} Q^{(j)}[\eta](\mathbf{h})\right\|_{2} \leqslant M\left|h_{l}\right|_{H^{s+\frac{1}{2}}} \prod_{k \neq l}\left|h_{k}\right|_{H^{s \vee t_{0}+1}}, \quad \text { for } 1 \leqslant l \leqslant j .
$$

Proposition 7. Let $t_{0}>\frac{1}{2}$ and $\eta \in H^{t_{0}+2}(\mathbb{R})$ be such that (8) is satisfied, and $k[\eta]$ be as defined in Proposition 2. For all $u \in \dot{H}^{\frac{1}{2}}(\mathbb{R})$, one has

$$
(u, \tilde{\mathcal{G}}[\eta] u) \leqslant M[\eta]|\mathfrak{B} u|_{2}^{2} \quad \text { and } \quad k[\eta]^{-1}|\mathfrak{B} u|_{2}^{2} \leqslant C(u, \tilde{\mathcal{G}}[\eta] u) .
$$


Proof. The first inequality can be obtained from (18) and Corollary 4. The proof of the second inequality is completely similar to Proposition 3.4 of [3], thus we omit the details here.

Proposition 8. Let $t_{0}>\frac{1}{2}, T>0$ and $\eta \in C^{1}\left([0, T] ; H^{t_{0}+2}(\mathbb{R})\right)$ be such that (8) is satisfied. Then, for all $u \in C^{1}\left([0, T] ; H^{\frac{1}{2}}(\mathbb{R})\right)$ and $t \in[0, T]$,

$$
\left|\left(\left[\partial_{t}, \tilde{\mathcal{G}}[\eta]\right] u(t), u(t)\right)\right| \leqslant M[\eta(t)]\left(\|\eta\|_{\infty, T}+\left\|\nabla \partial_{t} \eta\right\|_{\infty, T}\right)|\mathfrak{B} u(t)|_{2}^{2} .
$$

Proof. First of all,

$$
\left(u,\left[\partial_{t}, \tilde{\mathcal{G}}[\eta]\right] u\right)=\partial_{t}(u, \tilde{\mathcal{G}}[\eta] u)-2\left(u, \tilde{\mathcal{G}}[\eta] \partial_{t} u\right) .
$$

Due to Green's identity, one gets

$$
\begin{aligned}
\left(u,\left[\partial_{t}, \tilde{\mathcal{G}}[\eta]\right] u\right)= & \partial_{t} \int_{\mathcal{S}}([r+b(1-r)]+Q[\eta]) \nabla u^{\mathrm{b}} \cdot \nabla u^{\mathrm{b}} \\
& -2 \int_{\mathcal{S}}([r+b(1-r)]+Q[\eta]) \nabla\left(\partial_{t} u\right)^{\mathrm{b}} \cdot \nabla u^{\mathrm{b}} \\
= & \int_{\mathcal{S}} \partial_{t} Q[\eta] \nabla u^{\mathrm{b}} \cdot \nabla u^{\mathrm{b}}-2 \int_{\mathcal{S}}([r+b(1-r)]+Q[\eta]) \nabla\left(\left(\partial_{t} u\right)^{\mathrm{b}}-\partial_{t} u^{\mathrm{b}}\right) \cdot \nabla u^{\mathrm{b}} .
\end{aligned}
$$

Thus,

$$
\begin{aligned}
\left|\left(u,\left[\partial_{t}, \tilde{\mathcal{G}}[\eta]\right] u\right)\right| \leqslant & \left\|\partial_{t} Q[\eta]\right\|_{\infty}\left\|\nabla u^{\mathrm{b}}\right\|_{2}^{2} \\
& +\left(1+\|Q[\eta]\|_{\infty}\right)\left\|\nabla\left(\left(\partial_{t} u\right)^{b}-\partial_{t} u^{b}\right)\right\|_{2}\left\|\nabla u^{b}\right\|_{2} .
\end{aligned}
$$

Consider the system

$$
\left\{\begin{array}{l}
\nabla \cdot([r+b(1-r)]+Q[\eta]) \nabla w=\nabla \cdot \mathbf{g}, \\
\left.w\right|_{r=1}=0, \quad-\left.(0,1) \cdot P[\eta] \nabla w\right|_{r=0}=-\left.(0,1) \cdot \mathbf{g}\right|_{r=0},
\end{array}\right.
$$

where $w:=\left(\partial_{t} u\right)^{b}-\partial_{t} u^{b}$ and $\mathbf{g}=\left[\partial_{t}, Q[\eta]\right] \nabla u^{b}$. It follows from Proposition 3 that

$$
\left\|\nabla\left(\left(\partial_{t} u\right)^{b}-\partial_{t} u^{b}\right)\right\|_{2} \leqslant\left\|\partial_{t} Q[\eta]\right\|_{\infty}\left\|\nabla u^{b}\right\|_{2} .
$$

Finally, one can get the proposition from Corollary 4 and Proposition 2.

Proposition 9. For all $\underline{V} \in H^{t_{0}+1}(\mathbb{R})$ and $u \in H^{\frac{1}{2}}(\mathbb{R})$, one has

$$
\left(\underline{V} \partial_{z} u, \tilde{\mathcal{G}}[\eta] u\right) \leqslant M[\eta]|\underline{V}|_{W^{1, \infty}}|\mathcal{B} u|_{2}^{2} .
$$


Proof. Green's identity gives

$$
\left(\underline{V} \partial_{z} u, \tilde{\mathcal{G}}[\eta] u\right)=\int_{\mathcal{S}}([r+(1-b) r]+Q[\eta]) \nabla u^{b} \cdot \nabla\left(\underline{V} \partial_{z} u^{b}\right),
$$

and hence

$$
\begin{aligned}
\left(\underline{V} \partial_{z} u, \tilde{\mathcal{G}}[\eta] u\right)= & \int_{\mathcal{S}}([r+(1-b) r]+Q[\eta]) \nabla u^{b} \cdot\left[\nabla, \underline{V} \partial_{z}\right] u^{b} \\
& +\int_{\mathcal{S}} \nabla u^{b} \cdot\left[Q[\eta], \underline{V} \partial_{z}\right] u^{b} \\
& +\int_{\mathcal{S}} \nabla u^{b} \cdot\left(\underline{V} \partial_{z}\right)([r+(1-b) r]+Q[\eta]) u^{b} .
\end{aligned}
$$

Integrating by parts yields

$$
\begin{aligned}
\int_{\mathcal{S}} \nabla u^{b} \cdot\left(\underline{V} \partial_{z}\right)([r+(1-b) r]+Q[\eta]) \nabla u^{b} \\
=-\int_{\mathcal{S}}\left(\partial_{z} \underline{V}+\underline{V} \partial_{z}\right) \nabla u^{b} \cdot([r+(1-b) r]+Q[\eta]) \nabla u^{b} \\
=-\int_{\mathcal{S}}\left(\partial_{z} \underline{V}\right) \nabla u^{b} \cdot([r+(1-b) r]+Q[\eta]) \nabla u^{b} \\
-\int_{\mathcal{S}}\left[\underline{V} \partial_{z}, \nabla\right] u^{b} \cdot([r+(1-b) r]+Q[\eta]) \nabla u^{b} \\
-\int_{\mathcal{S}} \nabla\left(\underline{V} \partial_{z} u^{b}\right) \cdot([r+(1-b) r]+Q[\eta]) \nabla u^{b} .
\end{aligned}
$$

From the above facts, one can obtain

$$
\begin{aligned}
\left(\underline{V} \partial_{z} u, \tilde{\mathcal{G}}[\eta] u\right)= & \int_{\mathcal{S}}([r+(1-b) r]+Q[\eta]) \nabla u^{b} \cdot\left[\nabla, \underline{V} \partial_{z}\right] u^{b} \\
& +\frac{1}{2} \int_{\mathcal{S}} \nabla u^{b} \cdot\left[Q[\eta], \underline{V} \partial_{z}\right] u^{b} \\
& -\frac{1}{2} \int_{\mathcal{S}}\left(\partial_{z} \underline{V}\right) \nabla u^{b} \cdot([r+(1-b) r]+Q[\eta]) \nabla u^{b},
\end{aligned}
$$

which implies 


$$
\left(\underline{V} \partial_{z} u, \tilde{\mathcal{G}}[\eta] u\right) \lesssim|\underline{V}|_{W^{1, \infty}}\left(1+\|Q[\eta]\|_{W^{1, \infty}}\right)\left\|\nabla u^{\mathrm{b}}\right\|_{2}^{2}
$$

From Corollary 4, one completes the proof of the proposition.

Proposition 10. Let $t_{0}>\frac{1}{2}, s \geqslant t_{0}$ and $\underline{\eta} \in H^{s+\frac{3}{2}}(\mathbb{R})$ be such that (8) is satisfied. For all $\psi \in$ $\dot{H}^{s+\frac{3}{2}}(\mathbb{R})$, the mapping

$$
\eta \mapsto \tilde{\mathcal{G}}[\eta] \psi \in H^{s+\frac{1}{2}}(\mathbb{R})
$$

is well-defined and differentiable in a neighborhood of $\underline{\eta}$ in $H^{s+\frac{3}{2}}(\mathbb{R})$, and

$$
\forall h \in H^{s+\frac{3}{2}}(\mathbb{R}), \quad d_{\underline{\eta}} \tilde{\mathcal{G}}(h) \psi=-\tilde{\mathcal{G}}[\underline{\eta}](h \underline{Z})-\partial_{z}(h(1+\underline{\eta}) \underline{V}),
$$

where

$$
\underline{Z}:=\frac{1}{1+\underline{\eta}_{z}^{2}}\left(\mathcal{G}[\underline{\eta}] \psi+\underline{\eta}_{z} \psi_{z}\right) \quad \text { and } \quad \underline{V}=\partial_{z} \psi-\underline{Z}_{\underline{\eta}} .
$$

Proof. Differentiating (10) with respect to $\eta$ yields

$$
\left\{\begin{array}{l}
\nabla \cdot P \nabla v=-\nabla \cdot d_{\underline{\eta}} P(h) \nabla \phi, \\
\left.v\right|_{r=1}=0,\left.\quad(0,1) \cdot P \nabla v\right|_{r=0}=-\left.(0,1) \cdot\left(d_{\underline{\eta}} P(h) \nabla \phi\right)\right|_{r=0},
\end{array}\right.
$$

where $v$ is the derivative of the solution to (10) with respect to $\eta$ in the direction $h$. Next we prove that the function $v^{\mathrm{b}}:=\frac{d_{\underline{\eta}} \sigma(h)}{1+\partial_{r} \sigma} \partial_{r} \phi$ solves

$$
\left\{\begin{array}{l}
\nabla \cdot P \nabla v=-\nabla \cdot d_{\underline{\eta}} P(h) \nabla \phi, \\
\left.v\right|_{r=1}=\left.\frac{h}{1-b+\underline{\eta}} \partial_{r} \phi\right|_{r=1},\left.\quad(0,1) \cdot P \nabla v\right|_{r=0}=-\left.(0,1) \cdot\left(d_{\underline{\eta}} P(h) \nabla \phi\right)\right|_{r=0} .
\end{array}\right.
$$

It is not difficult to check that

$$
\nabla \cdot P \nabla v^{b}=\nabla \cdot \tilde{P} \nabla \phi
$$

where

$$
\left.\tilde{P}=-\partial_{r}\left(\begin{array}{ll}
\frac{d_{\underline{\eta}} \sigma(h)}{1+\partial_{r} \sigma} P
\end{array}\right)+\left(\begin{array}{ll}
0_{2 \times 1} & P \nabla\left(\frac{d_{\underline{\eta}} \sigma(h)}{1+\partial_{r} \sigma}\right)
\end{array}\right)+\left(\begin{array}{c}
0_{1 \times 2} \\
\left(P \nabla\left(\frac{d_{\underline{\eta}} \sigma(h)}{1+\partial_{r} \sigma}\right)\right.
\end{array}\right)^{\top}\right) .
$$

Noticing that

$$
P=(r+\sigma)\left(\begin{array}{cc}
1+\partial_{r} \sigma & -\partial_{z} \sigma \\
-\partial_{z} \sigma & \frac{1+\left(\partial_{z} \sigma\right)^{2}}{1+\partial_{r} \sigma}
\end{array}\right)
$$


one can obtain the expression of $d_{\underline{\eta}} P(h)$ as

$$
\begin{gathered}
d_{\underline{\eta}} \sigma(h)\left(\begin{array}{cc}
1+\partial_{r} \sigma & -\partial_{z} \sigma \\
-\partial_{z} \sigma & \frac{1+\left(\partial_{z} \sigma\right)^{2}}{1+\partial_{r} \sigma}
\end{array}\right) \\
+(r+\sigma)\left(\begin{array}{cc}
\partial_{r}\left(d_{\underline{\eta}} \sigma(h)\right) & -\partial_{z}\left(d_{\underline{\eta}} \sigma(h)\right) \\
-\partial_{z}\left(d_{\underline{\eta}} \sigma(h)\right) & \frac{2\left(\partial_{z} \sigma\right) \partial_{z}\left(d_{\underline{\eta}} \sigma(h)\right)}{1+\partial_{r} \sigma}-\frac{\partial_{r}\left(d_{\underline{\eta}} \sigma(h)\right)}{1+\partial_{r} \sigma} \frac{1+\left(\partial_{z} \sigma\right)^{2}}{1+\partial_{r} \sigma}
\end{array}\right) .
\end{gathered}
$$

A straightforward calculation yields

$$
\tilde{P}=-d_{\underline{\eta}} P(h) \text {. }
$$

Upon noting that $\left.d_{\underline{\eta}} \sigma(h)\right|_{r=1}=h$ and $\left.\partial_{r} \sigma\right|_{r=1}=\underline{\eta}-b$, one has

$$
\left.v^{b}\right|_{r=1}=\left.\frac{h}{1-b+\underline{\eta}} \partial_{r} \phi\right|_{r=1}
$$

On the other hand, it follows from $\left.d_{\underline{\eta}} \sigma(h)\right|_{r=0}=0$ that

$$
\left.(0,1) \cdot P \nabla v^{b}\right|_{r=0}=\left.\frac{\partial_{r}\left(d_{\underline{\eta}} \sigma(h)\right)}{\left(1+\partial_{r} \sigma\right)^{2}}(r+\sigma)\left(1+\left(\partial_{z} \sigma\right)^{2}\right) \partial_{r} \phi\right|_{r=0}=-\left.(0,1) \cdot\left(d_{\underline{\eta}} P(h) \nabla \phi\right)\right|_{r=0} .
$$

Hence $v^{b}$ solves (19) and therefore $v-v^{b}$ solves

$$
\left\{\begin{array}{l}
\nabla \cdot P \nabla\left(v-v^{b}\right)=0, \\
\left.\left(v-v^{b}\right)\right|_{r=1}=-\left.\frac{h}{1-b+\underline{\underline{\eta}}} \partial_{r} \phi\right|_{r=1},\left.\quad(0,1) \cdot P \nabla\left(v-v^{b}\right)\right|_{r=0}=0 .
\end{array}\right.
$$

The definition of the Dirichlet-Neumann operator $\tilde{\mathcal{G}}[\underline{\eta}]$ implies

$$
\left.(0,1) \cdot P \nabla\left(v-v^{b}\right)\right|_{r=1}=\tilde{\mathcal{G}}[\underline{\eta}]\left(-\left.\frac{h}{1-b+\underline{\eta}} \partial_{r} \phi\right|_{r=1}\right) .
$$

Noting that $\tilde{\mathcal{G}}[\underline{\eta}] \psi=\left.(0,1) \cdot P \nabla \phi\right|_{r=1}$, one has

$$
d_{\underline{\eta}} \tilde{\mathcal{G}}(h) \psi=\left.(0,1) \cdot d_{\underline{\eta}} P(h) \nabla \phi\right|_{r=1}+\left.(0,1) \cdot P \nabla v\right|_{r=1} \text {. }
$$

Since $\left.\partial_{r} \phi\right|_{r=1}=(1+\underline{\eta}-b) \underline{Z}$, the following identity holds

$$
d_{\underline{\eta}} \tilde{\mathcal{G}}(h) \psi=\left.(0,1) \cdot d_{\underline{\eta}} P(h) \nabla \phi\right|_{r=1}+\left.(0,1) \cdot P \nabla v^{b}\right|_{r=1}-\tilde{\mathcal{G}}[\underline{\eta}](h \underline{Z}) .
$$


Noting that

$$
\begin{aligned}
\partial_{n} v^{b}= & (0,1) \cdot\left(P \nabla\left(\frac{d_{\underline{\eta}} \sigma(h)}{1+\partial_{r} \sigma} \partial_{r} \phi\right)\right) \\
= & P_{21} \partial_{z}\left(\frac{d_{\underline{\eta}} \sigma(h)}{1+\partial_{r} \sigma} \partial_{r} \phi\right)+P_{22} \partial_{r}\left(\frac{d_{\underline{\eta}} \sigma(h)}{1+\partial_{r} \sigma} \partial_{r} \phi\right) \\
= & P_{21}\left[\partial_{z}\left(\frac{d_{\underline{\eta}} \sigma(h)}{1+\partial_{r} \sigma}\right) \partial_{r} \phi+\left(\frac{d_{\underline{\eta}} \sigma(h)}{1+\partial_{r} \sigma}\right) \partial_{z} \partial_{r} \phi\right] \\
& +P_{22}\left[\partial_{r}\left(\frac{d_{\underline{\eta}} \sigma(h)}{1+\partial_{r} \sigma}\right) \partial_{r} \phi+\left(\frac{d_{\underline{\eta}} \sigma(h)}{1+\partial_{r} \sigma}\right) \partial_{r} \partial_{r} \phi\right]
\end{aligned}
$$

and

$$
P_{22} \partial_{r} \partial_{r} \phi=-\partial_{r} P_{22} \partial_{r} \phi-\partial_{z}\left(P_{11} \partial_{z} \phi\right)-\partial_{z}\left(P_{12} \partial_{r} \phi\right)-\partial_{r}\left(P_{21} \partial_{z} \phi\right)
$$

one can get

$$
\begin{aligned}
\left.P_{22} \partial_{r} \partial_{r} \phi\right|_{r=1}= & -\left(1+\underline{\eta}_{z}^{2}\right)(1-b+\underline{\eta}) \underline{Z}-2(1+\underline{\eta}) \eta_{z}^{2} \underline{Z} \\
& -\partial_{z}\left((1+\underline{\eta}-b)(1+\underline{\eta}) \psi_{z}\right)+\partial_{z}\left(\underline{\eta}_{z}(1+\eta)(1-b+\underline{\eta}) \underline{Z}\right) \\
& +\underline{\eta}_{z}(2-b+\underline{2}) \psi_{z}+\underline{\eta}_{z}(1+\underline{\eta}) \partial_{z}((1-b+\underline{\eta}) \underline{Z}) \\
= & -(1-b+\underline{\eta}) \underline{Z}-(1+\underline{\eta}-b)(1+\underline{\eta}) \partial_{z} \psi_{z} \\
& +(1+\underline{\eta})(1-b+\underline{\eta}) \partial_{z}\left(\underline{\eta}_{z} \underline{Z}\right)+\underline{\eta}_{z}(1+\underline{\eta})(1-b+\underline{\eta}) \partial_{z} \underline{Z},
\end{aligned}
$$

and therefore

$$
\begin{aligned}
\left.(0,1) \cdot P \nabla v^{b}\right|_{r=1}= & -(1+\underline{\eta}) \underline{\eta}_{z} h_{z} \underline{Z}+\frac{(1+\underline{\eta})\left(1+\underline{\eta}_{z}^{2}\right)}{1-b+\underline{\eta}} h \underline{Z}-h \underline{Z} \\
& -(1+\underline{\eta}) h \partial_{z} \psi_{z}+(1+\underline{\eta}) h \partial_{z}\left(\underline{\eta}_{z} \underline{Z}\right) \\
= & -(1+\underline{\eta}) h_{z} \underline{\eta}_{z} \underline{Z}+\frac{(1+\underline{\eta})\left(1+\underline{\eta}_{z}^{2}\right)}{1-b+\underline{\eta}} h \underline{Z}-h \underline{Z} \\
& -(1+\underline{\eta}) h \partial_{z} \underline{V} .
\end{aligned}
$$

On the other hand, 


$$
\begin{aligned}
\left.(0,1) \cdot d_{\underline{\eta}} P(h) \nabla \phi\right|_{r=1}= & -\partial_{z}((1+\underline{\eta}) h) \psi_{z}+\left(1+\underline{\eta}_{z}^{2}\right) h \underline{Z} \\
& +2(1+\underline{\eta}) h_{z} \underline{\eta}_{z} \underline{Z}-\frac{(1+\underline{\eta})\left(1+\underline{\eta}_{z}^{2}\right)}{1-b+\underline{\eta}} h \underline{Z} \\
= & (1+\underline{\eta}) h_{z} \underline{\eta}_{z} \underline{Z}-\frac{(1+\underline{\eta})\left(1+\underline{\eta}_{z}^{2}\right)}{1-b+\underline{\eta}} h \underline{Z}+h \underline{Z} \\
& -\partial_{z}((1+\underline{\eta}) h) \underline{V} .
\end{aligned}
$$

Hence, one has

$$
\left.(0,1) \cdot d_{\underline{\eta}} P(h) \nabla \phi\right|_{r=1}+\left.(0,1) \cdot P \nabla v^{b}\right|_{r=1}=-\partial_{z}((1+\underline{\eta}) h \underline{V}),
$$

which implies the proposition.

\section{Linearization formulas}

In this section, we give the linearization formulas. For all $\alpha=\left(\alpha_{0}, \alpha_{1}\right)^{\top} \in \mathbb{N}^{1+1}$, we denote by $\partial^{\alpha_{0}}$ the time derivatives and $\partial^{\alpha_{1}}$ the spatial derivatives. Let $\eta_{(\alpha)}=\partial^{\alpha} \eta, \psi_{(\alpha)}=\partial^{\alpha} \psi-\underline{Z} \partial^{\alpha} \eta$ with $\underline{Z}=\frac{\mathcal{G} \psi+\eta_{z} \psi_{z}}{1+\eta_{z}^{2}}$ and $\underline{V}=\psi_{z}-\underline{Z} \eta_{z}$. We introduce the energy $\mathcal{E}^{N}(U)$ as follows

$$
\mathcal{E}^{N}(U)=\left\|\psi_{z}\right\|_{H^{t_{0}+2}}^{2}+\sum_{\alpha \in \mathbb{N}^{1+1},|\alpha| \leqslant N}\left(\left\|\eta_{(\alpha)}\right\|_{H^{1}}^{2}+\left\|\mathfrak{B} \psi_{(\alpha)}\right\|_{2}^{2}\right) .
$$

For all $T>0$, we denote by $E_{T}^{N}$ the functional space

$$
E_{T}^{N}=\left\{U \in C\left([0, T] ; H^{t_{0}+2} \times \dot{H}^{2}(\mathbb{R})\right), \sup _{0 \leqslant t \leqslant T} \mathcal{E}^{N}(U(t))<\infty\right\}
$$

Denoting $\mathfrak{m}^{N}(U)=C\left(M[\eta(t)], \mathcal{E}^{N}(U(t))\right)$, we have the following linearization formula.

Proposition 11. Let $t_{0}>\frac{1}{2}$ and $N \in \mathbb{N}$. Moreover, assume that $N \geqslant t_{0}+t_{0} \vee 2+\frac{3}{2}$ and $U=$ $(\eta, \psi)^{\top}$ is such that $\mathcal{E}^{N}(U)<\infty$ and satisfy (8). Then for all $\alpha \in \mathbb{N}^{1+1}$ with $1 \leqslant|\alpha| \leqslant N$, one has

$$
\begin{array}{ll}
\partial^{\alpha}(\mathcal{G} \psi)=\mathcal{G} \psi_{(\alpha)}+R_{\alpha}, & |\alpha| \leqslant N-1, \\
\partial^{\alpha}(\mathcal{G} \psi)=\mathcal{G} \psi_{(\alpha)}-\partial_{z}\left(\underline{V} \eta_{(\alpha)}\right)+\mathcal{G}_{(\alpha)} \psi_{\langle\check{\alpha}\rangle}+R_{\alpha}, & |\alpha|=N,
\end{array}
$$

where $\eta_{(\alpha)}=\partial^{\alpha} \eta, \psi_{(\alpha)}=\partial^{\alpha} \psi-\underline{Z} \partial^{\alpha} \eta$ with $\underline{Z}=\frac{\mathcal{G} \psi+\eta_{z} \psi_{z}}{1+\eta_{z}^{2}}, \underline{V}=\psi_{z}-\underline{Z} \eta_{z}, \psi_{\langle\check{\alpha}\rangle}=\left(\psi_{\left(\check{\alpha}^{0}\right)}, \psi_{\left(\check{\alpha}^{1}\right)}\right)$ with $\check{\alpha}^{j}=\alpha-\mathbf{e}_{j}$,

$$
\mathcal{G}_{(\alpha)} \psi_{\langle\check{\alpha}\rangle}:=\frac{1}{1+\eta} \tilde{\mathcal{G}}_{(\alpha)} \psi_{\langle\check{\alpha}\rangle}=\frac{1}{1+\eta} \sum_{j=0}^{1} \alpha_{j} d \tilde{\mathcal{G}}\left(\partial_{j} \eta\right) \psi_{\left(\check{\alpha}^{j}\right)},
$$


and $R_{\alpha}$ satisfies

$$
\left|R_{\alpha}\right|_{H^{1}} \leqslant \mathfrak{m}^{N}(U)
$$

Proof. It is easy to know that

$$
\left|\partial^{\alpha} \mathcal{G} \psi-\frac{\partial^{\alpha} \tilde{\mathcal{G}} \psi}{1+\eta}\right|_{H^{1}} \leqslant \mathfrak{m}^{N}(U)
$$

On the other hand, one has

$$
\partial^{\alpha} \tilde{\mathcal{G}} \psi=\tilde{\mathcal{G}} \partial^{\alpha} \psi+d \tilde{\mathcal{G}}\left(\partial^{\alpha} \eta\right) \psi+\tilde{\mathcal{G}}_{(\alpha)} \psi_{\langle\check{\alpha}\rangle}+\tilde{R}_{\alpha},
$$

with

$$
\tilde{R}_{\alpha}=\left(\sum_{j=0}^{1} \alpha_{j} d \tilde{\mathcal{G}}\left(\partial^{\alpha} \eta\right) \psi-\tilde{\mathcal{G}}_{(\alpha)} \psi_{\langle\check{\alpha}\rangle}\right)+R_{\alpha}^{\prime}
$$

and $R_{\alpha}^{\prime}$ is a sum of terms of the form

$$
d^{j} \tilde{\mathcal{G}}\left(\partial^{\iota^{1}} \eta, \cdots, \partial^{\iota^{j}} \eta\right) \partial^{\delta} \psi
$$

where $j \in \mathbb{N}, \iota=\left(\iota^{1}, \cdots, \iota^{j}\right) \in \mathbb{N}^{j}$ and $\delta \in \mathbb{N}$ satisfy

$$
\sum_{i=1}^{j}\left|\iota^{i}\right|+|\delta|=N, \quad 0 \leqslant|\delta| \leqslant N-2 \quad \text { and } \quad\left|\iota^{i}\right|<N
$$

Applying the same arguments of Proposition 9.3 in [16] and utilizing Proposition 6, one can get

$$
\left|\tilde{R}_{\alpha}\right|_{H^{1}} \leqslant \mathfrak{m}^{N}(U) .
$$

On the other hand, it is easy to see that

$$
\left|\frac{1}{1+\eta} \partial_{z}\left(\underline{V}(1+\eta) \eta_{(\alpha)}\right)-\partial_{z}\left(\underline{V} \eta_{(\alpha)}\right)\right|_{H^{1}} \leqslant \mathfrak{m}^{N}(U) .
$$

Finally, one can deduce Proposition 11 from the above facts and Proposition 10.

Lemma 12. Let $t_{0}>\frac{1}{2}$ and $N \in \mathbb{N}$. Moreover, assume that $N \geqslant t_{0}+t_{0} \vee 2+\frac{3}{2}$ and $U=(\eta, \psi)^{\top}$ satisfies (8) and $\mathcal{E}^{N}(U)<\infty$. Letting $\alpha=\beta+\delta$ with $|\delta|=1$, for all $\alpha \in \mathbb{N}^{1+1}$ with $1 \leqslant|\alpha| \leqslant N$, one has

$$
\begin{aligned}
\partial^{\beta}\left(\underline{V} \partial_{z} \eta\left(\partial_{z} \partial^{\delta} \psi-\underline{Z} \partial_{z} \partial^{\delta} \eta\right)\right) & \sim \underline{V} \partial_{z} \psi(\alpha)+\left(\underline{V} \partial_{z} \underline{Z}\right) \partial^{\alpha} \eta-\underline{V}\left\{\partial^{\beta}, \underline{Z}\right\} \partial_{z} \partial^{\delta} \eta, \\
\partial^{\beta}\left(\underline{Z} \partial^{\delta}(\mathcal{G} \psi)\right) & \sim \underline{Z} \partial^{\alpha}(\mathcal{G} \psi)+\left\{\partial^{\beta}, \underline{Z}\right\} \partial^{\delta}(\mathcal{G} \psi),
\end{aligned}
$$


where $\left\{\partial^{\beta}, \underline{w}\right\}=\sum_{j=0}^{1} \beta_{j} \partial_{j} \underline{w} \partial^{\check{\beta}^{j}}$ with $\check{\beta}^{j}=\beta-e_{j}$, and $a \sim b$ means that

$$
|\mathfrak{B}(a-b)|_{2} \leqslant \mathfrak{m}^{N}(U) .
$$

Proof. Adopting the same arguments of Lemma 4.15 in [16] or Lemma 9 in [17], one can obtain the lemma from Propositions 6 and 10.

\section{Quasilinearization}

Let

$$
\mathcal{A}[U]=\left(\begin{array}{cc}
0 & -\mathcal{G}[\eta] \\
\mathfrak{a}-\frac{1}{1+\eta} \partial_{z}\left(\tilde{\mathcal{K}}\left[\eta_{z}\right] \partial_{z} \bullet\right) & 0
\end{array}\right), \quad \mathcal{B}[U]=\left(\begin{array}{cc}
\partial_{z}(\underline{V} \bullet) & 0 \\
0 & \underline{V} \partial_{z}
\end{array}\right)
$$

and

$$
\mathcal{C}_{\alpha}[U]=\left(\begin{array}{cc}
0 & -\frac{1}{1+\eta} \tilde{\mathcal{G}}_{(\alpha)} \\
\mathcal{K}_{(\alpha)}\left[\eta_{z}\right] & 0
\end{array}\right)
$$

where

$$
\mathfrak{a}=\frac{J}{2 \pi(1+\eta)^{2}} m_{1}\left(\frac{J}{2 \pi(1+\eta)}\right)+\left(\partial_{t}+\underline{V} \partial_{z}\right) \underline{Z}
$$

and

$$
\tilde{\mathcal{K}}\left[\eta_{z}\right]=\frac{1+\eta}{\left(1+\eta_{z}^{2}\right)^{\frac{3}{2}}}
$$

and for any $F=\left(f_{0}, f_{1}\right)^{\top}$,

$$
\mathcal{K}_{(\alpha)}\left[\eta_{z}\right] F=-\partial_{z}\left[\sum_{j=0}^{1}\left(d \mathcal{K}\left(\partial_{j} \eta_{z}\right) \partial_{z} f_{j}+d \mathcal{K}\left(\partial_{z} f_{j}\right) \partial_{j} \eta_{z}\right)\right] .
$$

One then has the following proposition.

Proposition 13. Let $t_{0}>\frac{1}{2}$ and $N \in \mathbb{N}$. Moreover, assume that $N \geqslant t_{0}+t_{0} \vee 2+\frac{3}{2}$ and $U=$ $(\eta, \psi)^{\top}$ satisfies (8) and $\mathcal{E}^{N}(U)<\infty$. Then

$$
\begin{array}{rlrl}
\partial_{t} U_{(\alpha)}+\mathcal{A}[U] U_{(\alpha)} & =\left(R_{\alpha}, S_{\alpha}\right)^{\top}, & |\alpha|<N, \\
\partial_{t} U_{(\alpha)}+\mathcal{A}[U] U_{(\alpha)}+\mathcal{B}[U] U_{(\alpha)}+\mathcal{C}_{\alpha} U_{\langle\check{\alpha}\rangle}=\left(R_{\alpha}, S_{\alpha}\right)^{\top}, & |\alpha|=N,
\end{array}
$$

where $U_{\langle\check{\alpha}\rangle}=\left(\eta_{\langle\check{\alpha}\rangle}, \psi_{\langle\check{\alpha}\rangle}\right)^{\top}$ and

$$
\left|R_{\alpha}\right|_{H^{1}}+\left|\mathcal{B} S_{\alpha}\right|_{2} \leqslant \mathfrak{m}^{N}(U)
$$


Proof. It follows from Proposition 11 that

$$
\partial_{t} \eta_{(\alpha)}-\mathcal{G} \psi_{(\alpha)}+\partial_{z}\left(\underline{V} \eta_{(\alpha)}\right)-\frac{1}{1+\eta} \tilde{\mathcal{G}}_{(\alpha)} \psi_{\langle\check{\alpha}\rangle}=R_{\alpha} .
$$

It is easy to check that

$$
\begin{aligned}
& \partial_{t} \partial^{\delta} \psi+\underline{V} \partial_{z} \eta\left(\partial_{z} \partial^{\delta} \psi-\underline{Z} \partial_{z} \partial^{\delta} \eta\right)-\underline{Z} \partial^{\delta}(\mathcal{G} \psi) \\
& +\partial^{\delta}\left(-v\left(\frac{J}{2 \pi(1+\eta)}\right)+v\left(\frac{J}{2 \pi}\right)+\kappa_{1}+\kappa_{2}\right)=0 .
\end{aligned}
$$

Following (9.17) of [16], one obtains

$$
\partial^{\alpha} \kappa_{1} \sim-\partial_{z}\left(\mathcal{K}\left[\eta_{z}\right] \partial_{z} \partial^{\alpha} \eta\right)+\mathcal{K}_{(\alpha)}\left[\eta_{z}\right] \eta_{\langle\check{\alpha}\rangle}
$$

where $\mathcal{K}\left[\eta_{z}\right]=\frac{1}{\left(1+\eta_{z}^{2}\right)^{\frac{3}{2}}}$. (26) can be rewritten as

$$
\partial^{\alpha} \kappa_{1} \sim-\frac{1}{1+\eta} \partial_{z}\left((1+\eta) \mathcal{K}\left[\eta_{z}\right] \partial_{z} \partial^{\alpha} \eta\right)+\frac{\eta_{z} \partial_{z} \partial^{\alpha} \eta}{(1+\eta)\left(1+\eta_{z}^{2}\right)^{\frac{3}{2}}}+\mathcal{K}_{(\alpha)}\left[\eta_{z}\right] \eta_{\langle\check{\alpha}\rangle}
$$

Noticing that

$$
\partial^{\alpha} \kappa_{2} \sim-\frac{\eta_{z} \partial_{z} \partial^{\alpha} \eta}{(1+\eta)\left(1+\eta_{z}^{2}\right)^{\frac{3}{2}}}
$$

one has

$$
\partial^{\alpha} \kappa \sim-\frac{1}{1+\eta} \partial_{z}\left(\tilde{\mathcal{K}}\left[\eta_{z}\right] \partial_{z} \partial^{\alpha} \eta\right)+\mathcal{K}_{(\alpha)}\left[\eta_{z}\right] \eta_{\langle\check{\alpha}\rangle}
$$

where $\tilde{\mathcal{K}}\left[\eta_{z}\right]=(1+\eta) \mathcal{K}\left[\eta_{z}\right]=\frac{1+\eta}{\left(1+\eta_{z}^{2}\right)^{\frac{3}{2}}}$. On the other hand,

$$
\begin{aligned}
\partial^{\alpha}\left(v\left(\frac{J}{2 \pi(1+\eta)}\right)-v\left(\frac{J}{2 \pi}\right)\right) & =-\frac{J}{2 \pi} \partial^{\beta}\left(m_{1}\left(\frac{J}{2 \pi(1+\eta)}\right) \frac{\partial^{\delta} \eta}{(1+\eta)^{2}}\right) \\
& \sim-\frac{J}{2 \pi(1+\eta)^{2}} m_{1}\left(\frac{J}{2 \pi(1+\eta)}\right) \partial^{\alpha} \eta .
\end{aligned}
$$

Since $\partial_{t} \eta_{(\alpha)}=\partial^{\alpha}(\mathcal{G} \psi)$, from (25)-(28) and Lemma 12, and noting that

$$
\underline{V}\left\{\partial^{\beta}, \underline{Z}\right\} \partial_{z} \partial^{\delta} \eta+\left\{\partial^{\beta}, \underline{Z}\right\} \partial^{\delta}(\mathcal{G} \psi) \sim\left\{\partial^{\beta}, \underline{Z}\right\}\left(\underline{V} \partial_{z} \partial^{\delta} \eta+\partial^{\delta}(\mathcal{G} \psi)\right) \sim 0,
$$

one can obtain

$$
\partial_{t} \psi_{(\alpha)}+\underline{V} \partial_{z} \psi_{(\alpha)}+\left(\mathfrak{a}-\frac{1}{1+\eta} \partial_{z}\left(\tilde{\mathcal{K}}\left[\eta_{z}\right] \partial_{z} \bullet\right)\right) \eta_{(\alpha)}+\mathcal{K}_{(\alpha)}\left[\eta_{z}\right] \eta_{\langle\check{\alpha}\rangle} \sim 0,
$$


where

$$
\mathfrak{a}=\frac{J}{2 \pi(1+\eta)^{2}} m_{1}\left(\frac{J}{2 \pi(1+\eta)}\right)+\left(\partial_{t}+\underline{V} \partial_{z}\right) \underline{Z} .
$$

Finally, we can finish the proof by using (24) and (29).

\section{Proof of Theorem 1}

We first discuss the initial condition for our problem, which is similar to that of the water-wave equations without magnetic field. Since $\mathcal{E}^{N}(U)$ involves time derivatives, we must specify in what sense the initial energy $\mathcal{E}^{N}\left(U^{0}\right)$ holds. We must choose initial values $U_{(\alpha)}^{0}$ for $\left.\left(U_{(\alpha)}\right)\right|_{t=0}$ when $\alpha_{0}>0$, in terms of $U^{0}$ and its spatial derivatives. Similar to [17], we achieve this via a finite induction. When $\alpha_{0}=0$, we take

$$
U_{(\alpha)}^{0}=\left(\partial^{\alpha} \eta^{0}, \partial^{\alpha} \psi^{0}-\underline{Z}^{0} \partial^{\alpha} \eta^{0}\right)^{\top}
$$

where $\underline{Z}^{0}=\frac{\mathcal{G} \psi^{0}+\eta_{z}^{0} \psi_{z}^{0}}{1+\left(\eta_{z}^{0}\right)^{2}}$. We assume that $\left.U_{(\beta)}\right|_{t=0}=U_{(\beta)}^{0}$ has been chosen for all $\beta=\left(\beta_{0}, \beta_{1}\right)^{\top} \in$ $\mathbb{N}^{d+1}$ with $\beta_{0}<n(1 \leqslant n \leqslant N)$. When $\alpha_{0}=n$, we choose

$$
\left.U_{(\alpha)}\right|_{t=0}=\left.\left(\partial_{t} \eta_{\left(\alpha^{\prime}\right)}, \partial_{t} \psi_{\left(\alpha^{\prime}\right)}+\partial_{t} \underline{Z} \partial^{\alpha^{\prime}} \eta\right)^{\top}\right|_{t=0},
$$

where $\alpha^{\prime}=\left(\alpha_{0}-1, \alpha_{1}\right)^{\top}$, and we therefore are led to set up initial conditions for $\partial_{t} U_{\left(\alpha^{\prime}\right)}$, which can be achieved by using Proposition 13. Next, we define the initial energy by

$$
\mathcal{E}^{N}\left(U^{0}\right)=\left\|\psi_{z}^{0}\right\|_{H^{t_{0}+2}}+\sum_{\alpha \in \mathbb{N}^{1+1},|\alpha| \leqslant N}\left(\left\|\eta_{(\alpha)}^{0}\right\|_{H^{1}}^{2}+\left\|\mathfrak{B} \psi_{(\alpha)}^{0}\right\|_{2}^{2}\right)
$$

with $U_{(\alpha)}^{0}$ constructed above.

\subsection{Mollified quasilinear system}

Let $\chi: \mathbb{R} \rightarrow \mathbb{R}$ be a smooth and compactly supported function, which equals one in a neighborhood of the origin. For all $0<\iota<1$, we denote by $J^{\iota}$ the mollifier $J^{\iota}=\chi\left(\iota\left|i \partial_{z}\right|\right)$. Consider the following system

$$
\left\{\begin{array}{l}
\partial_{t} \eta-J^{\iota} \mathcal{G} \psi=0 \\
\partial_{t} \psi+\frac{1}{2} J^{\iota}\left(\psi_{z}^{2}-\frac{\left(\mathcal{G} \psi+\eta_{z} \psi_{z}\right)^{2}}{1+\eta_{z}^{2}}\right)+J^{\iota}\left(-v\left(\frac{J}{2 \pi(1+\eta)}\right)+v\left(\frac{J}{2 \pi}\right)+\kappa\right)=0
\end{array}\right.
$$

Since $J^{\iota}$ is a smoothing operator, from the Cauchy-Lipschitz theorem of ODE, we know (31) has a unique maximal solution $U^{\iota}=\left(\eta^{\iota}, \psi^{\iota}\right)^{\top}$ with the initial data $\left(\eta^{0}, \psi^{0}\right)^{\top}$ on a time interval $\left[0, T_{\text {max }}^{\iota}\right]$. For $\alpha \in \mathbb{N}^{1+1}$ and $1 \leqslant|\alpha| \leqslant N$, if we set $U_{(\alpha)}^{\iota}=\left(\eta_{(\alpha)}^{\iota}, \psi_{(\alpha)}^{\iota}\right)^{\top}$, where

$$
\eta_{(\alpha)}^{\iota}=\partial^{\alpha} \eta^{\iota}, \quad \psi_{(\alpha)}^{\iota}=\partial^{\alpha} \psi^{\iota}-\underline{Z}^{\iota} \partial^{\alpha} \eta^{\iota},
$$


and

$$
\underline{Z}^{\iota}=\frac{\mathcal{G}\left[\eta^{\iota}\right] \psi^{\iota}+\eta_{z}^{\iota} \psi_{z}^{\iota}}{1+\left(\eta_{z}^{\iota}\right)^{2}},
$$

then it is easy to check that

$$
\partial_{t} U_{(\alpha)}^{\iota}+J^{\iota}\left(\mathcal{A}\left[U^{\iota}\right]+\mathcal{B}\left[U^{\iota}\right]\right) U_{(\alpha)}^{\iota}+J^{\iota} \mathcal{C}_{\alpha}\left[U^{\iota}\right] U_{\langle\check{\alpha}\rangle}^{\iota}=\left(J^{\iota} R_{\alpha}, J^{\iota} S_{\alpha}+S_{\alpha}^{\prime}\right)^{\top},
$$

where $S_{\alpha}^{\prime}=-\left(1-J^{\iota}\right)\left[\eta_{(\alpha)}^{\iota} \partial_{t} \underline{Z}^{\iota}\right]+\left[\underline{Z}^{\iota}, J^{\iota}\right] \partial^{\alpha}\left(\mathcal{G} \psi^{\iota}\right)$.

\subsection{Symmetrizer and energy}

We denote a symmetrizer of system (32) by

$$
\mathcal{S}[U]=\mathcal{S}^{1}[U]+\mathcal{S}_{\alpha}^{2}[U],
$$

where

$$
\begin{aligned}
& \mathcal{S}^{1}[U]=\operatorname{diag}\left(\left((1+\eta) \mathfrak{a}-\partial_{z}\left(\tilde{\mathcal{K}}\left[\eta_{z}\right] \partial_{z} \bullet\right)\right), \tilde{\mathcal{G}}[\eta]\right), \\
& \mathcal{S}_{\alpha}^{2}[U]=\operatorname{diag}\left((1+\eta) \mathcal{K}_{(\alpha)}\left[\eta_{z}\right] \bullet, \tilde{\mathcal{G}}_{(\alpha)}\right) .
\end{aligned}
$$

The energy $\mathcal{F}^{l}(U)$ can be defined as

$$
\mathcal{F}^{l}(U)=\sum_{0 \leqslant|\alpha| \leqslant l} \mathcal{F}^{\alpha}(U),
$$

for all $1 \leqslant l \leqslant N$, where

$$
\begin{array}{ll}
\mathcal{F}^{\alpha}(U)=\frac{1}{2}\left(\mathcal{S}^{1}[U] U_{(\alpha)}, U_{(\alpha)}\right), & \\
\mathcal{F}^{0}(U)=M[\eta]\left(|\eta|_{H^{1}}^{2}+(\psi, \tilde{\mathcal{G}}[0] \psi)\right), & \text { if } \alpha=0,
\end{array}
$$

for $U_{(\alpha)}=\left(\eta_{(\alpha)}, \psi_{(\alpha)}\right)$. The following lemma implies that $\mathcal{F}^{j}(U)$ is equivalent to the energy $\mathcal{E}^{j}(U)$.

Lemma 14. Assuming $U$ solves (4) on $[0, T]$ and satisfies (8), for all $0 \leqslant j \leqslant N$, we have

$$
\mathcal{E}^{j}(U) \leqslant M[\eta] \mathcal{F}^{j}(U) \text { and } \mathcal{F}^{j}(U) \leqslant M[\eta] \mathcal{E}^{j}(U)
$$

Proof. The definition of $\mathcal{F}^{j}(U)$ implies

$$
\begin{aligned}
\mathcal{F}^{j}(U)= & \sum_{\leqslant|\alpha| \leqslant j}\left[\left((1+\eta) \mathfrak{a} \eta_{(\alpha)}-\partial_{z} \tilde{\mathcal{K}}\left[\eta_{z}\right] \partial_{z} \eta_{(\alpha)}, \eta_{(\alpha)}\right)+\left(\tilde{\mathcal{G}}[\eta] \psi_{(\alpha)}, \psi_{(\alpha)}\right)\right] \\
& +M[\eta]\left(|\eta|_{H^{1}}^{2}+(\psi, \tilde{\mathcal{G}}[0] \psi)\right) .
\end{aligned}
$$


Firstly, it is easy to check that

$$
M^{-1}[\eta]\left|\partial_{z} \eta_{(\alpha)}\right|_{2}^{2} \leqslant\left(\tilde{\mathcal{K}}\left[\eta_{z}\right] \partial_{z} \eta_{(\alpha)}, \partial_{z} \eta_{(\alpha)}\right) \leqslant M[\eta]\left|\partial_{z} \eta_{(\alpha)}\right|_{2}^{2}
$$

and

$$
\left|\left((1+\eta) \mathfrak{a} \eta_{(\alpha)}, \eta_{(\alpha)}\right)\right| \leq \frac{1}{2} M^{-1}[\eta]\left|\partial_{z} \eta_{(\alpha)}\right|_{2}^{2}+\frac{1}{2} M[\eta]|\eta|_{H^{1}}^{2}
$$

On the other hand, from Proposition 7

$$
M^{-1}[\eta]\left\|\mathfrak{B} \psi_{(\alpha)}\right\|_{2}^{2} \leqslant\left(\tilde{\mathcal{G}}[\eta] \psi_{(\alpha)}, \psi_{(\alpha)}\right) \leqslant M[\eta]\left\|\mathfrak{B} \psi_{(\alpha)}\right\|_{2}^{2} .
$$

Thus, one obtains (33).

\subsection{Energy estimates}

Taking the $L^{2}$-scalar product of (32) with $\mathcal{S}^{1} U_{(\alpha)}^{\iota}+\mathcal{S}_{\alpha}^{2} U_{\langle\check{\alpha}\rangle}^{\iota}$, and noting that

$$
\left(J^{\iota} \mathcal{A} U_{(\alpha)}, \mathcal{S}^{1} U_{(\alpha)}\right)=0
$$

and

$$
\left(J^{\iota} \mathcal{A} U_{(\alpha)}, \mathcal{S}_{\alpha}^{2} U_{\langle\check{\alpha}\rangle}\right)+\left(J^{\iota} \mathcal{C}_{\alpha} U_{\langle\check{\alpha}\rangle}, \mathcal{S}^{1} U_{(\alpha)}\right)=0,
$$

one can get

$$
\frac{d}{d t}\left(\mathcal{F}^{\alpha}\left(U^{\iota}\right)+\left(U_{(\alpha)}^{\iota}, \mathcal{S}_{\alpha}^{2} U_{\langle\breve{\alpha}\rangle}^{\iota}\right)\right)=\sum_{j=1}^{5} A_{j}+B_{1}+B_{2},
$$

where

$$
\begin{array}{ll}
A_{1}=\frac{1}{2}\left(\left[\partial_{t}, \mathcal{S}^{1}\right] U_{(\alpha)}^{\iota}, U_{(\alpha)}^{\iota}\right), & A_{2}=-\left(J^{\iota} \mathcal{B} U_{(\alpha)}^{\iota}, \mathcal{S}^{1} U_{(\alpha)}^{\iota}\right), \\
A_{3}=\left(U_{(\alpha)}, \partial_{t}\left(\mathcal{S}_{\alpha}^{2} U_{\langle\breve{\alpha}\rangle}^{\iota}\right)\right), & A_{4}=-\left(J^{\iota} \mathcal{B} U_{(\alpha)}^{\iota}, \mathcal{S}_{\alpha}^{2} U_{\langle\check{\alpha}\rangle}^{\iota}\right), \\
A_{5}=-\left(J^{\iota} \mathcal{C}_{\alpha} U_{\langle\check{\alpha}\rangle}^{\iota}, \mathcal{S}_{\alpha}^{2} U_{\langle\check{\alpha}\rangle}^{\iota}\right) &
\end{array}
$$

and

$$
B_{1}=\left(\left(R_{\alpha}, S_{\alpha}\right)^{\top}, \mathcal{S}^{1} U_{(\alpha)}^{\iota}+\mathcal{S}_{\alpha}^{2} U_{\langle\check{\alpha}\rangle}^{\iota}\right), \quad B_{2}=\left(\left(0, S_{\alpha}^{\prime}\right)^{\top}, \mathcal{S}^{1} U_{(\alpha)}^{\iota}+\mathcal{S}_{\alpha}^{2} U_{\langle\check{\alpha}\rangle}^{\iota}\right)
$$

Estimate of $A_{1}$. First, we have

$$
2 A_{1}=\left(\left(\partial_{t}((1+\eta) \mathfrak{a}) \eta_{(\alpha)}, \eta_{(\alpha)}\right)\right)+\left(\left[\partial_{t}, \tilde{\mathcal{G}}\right] \psi_{(\alpha)}, \psi_{(\alpha)}\right)+\left(\partial_{t} \tilde{\mathcal{K}} \partial_{z} \eta_{(\alpha)}, \partial_{z} \eta_{(\alpha)}\right)
$$


where we denote $\left(\eta_{\alpha}^{\iota}, \psi_{(\alpha)}^{\iota}\right)$ as $\left(\eta_{(\alpha)}, \psi_{(\alpha)}\right)$ for simplicity. It is easy to get that

$$
\left|\left(\left(\partial_{t}((1+\eta) \mathfrak{a}) \eta_{(\alpha)}, \eta_{(\alpha)}\right)\right)\right| \leqslant \mathfrak{m}^{N}(U),
$$

and

$$
\left|\left(\partial_{t} \tilde{\mathcal{K}} \partial_{z} \eta_{(\alpha)}, \partial_{z} \eta_{(\alpha)}\right)\right| \leqslant \mathfrak{m}^{N}(U) .
$$

From Proposition 8 one obtains

$$
\left|\left(\left[\partial_{t}, \tilde{\mathcal{G}}\right] \psi_{(\alpha)}, \psi_{(\alpha)}\right)\right| \leqslant \mathfrak{m}^{N}(U) .
$$

Hence, we have $\left|A_{1}\right| \leqslant \mathfrak{m}^{N}(U)$.

Estimate of $A_{2}$. One has

$$
\begin{aligned}
A_{2}= & -\left((1+\eta) \mathfrak{a} \eta_{(\alpha)}, J^{\iota} \partial_{z}\left(\underline{V} \eta_{(\alpha)}\right)\right)+\left(\partial_{z}\left(\tilde{\mathcal{K}} \partial_{z} \eta_{(\alpha)}\right), J^{\iota} \partial_{z}\left(\underline{V} \eta_{(\alpha)}\right)\right) \\
& -\left(\tilde{\mathcal{G}} \psi_{(\alpha)}, J^{\iota}\left(\underline{V} \partial_{z} \psi_{(\alpha)}\right)\right)
\end{aligned}
$$

It is easy to get

$$
\begin{aligned}
\left(\partial_{z}\left(\tilde{\mathcal{K}} \partial_{z} \eta_{(\alpha)}\right), J^{\iota} \partial_{z}\left(\underline{V} \eta_{(\alpha)}\right)\right) & \sim\left(\partial_{z}\left(\tilde{\mathcal{K}} \partial_{z} \eta_{(\alpha)}\right), J^{\iota}\left(\underline{V} \partial_{z} \eta_{(\alpha)}\right)\right) \\
& \sim\left(\underline{V} \partial_{z}\left(\tilde{\mathcal{K}} \partial_{z} \eta_{(\alpha)}\right), J^{\iota} \partial_{z} \eta_{(\alpha)}\right) \\
& \sim\left(\partial_{z}\left(\tilde{\mathcal{K}} \partial_{z}\left(\underline{V} \eta_{(\alpha)}\right)\right), J^{\iota} \partial_{z} \eta_{(\alpha)}\right) \\
& \left.=-\left(\partial_{z}\left(\underline{V} \eta_{(\alpha)}\right)\right), \tilde{\mathcal{K}} \partial_{z} J^{\iota} \partial_{z} \eta_{(\alpha)}\right) \\
& \left.\sim-\left(\partial_{z}\left(\underline{V} \eta_{(\alpha)}\right)\right), J^{\iota}\left(\partial_{z}\left(\tilde{\mathcal{K}} \partial_{z} \eta_{(\alpha)}\right)\right)\right) \\
& =\left(\partial_{z}\left(\tilde{\mathcal{K}} \partial_{z} \eta_{(\alpha)}\right), J^{\iota} \partial_{z}\left(\underline{V} \eta_{(\alpha)}\right)\right)
\end{aligned}
$$

which implies

$$
\left(\partial_{z}\left(\tilde{\mathcal{K}} \partial_{z} \eta_{(\alpha)}\right), J^{\iota} \partial_{z}\left(\underline{V} \eta_{(\alpha)}\right)\right) \sim 0
$$

where $a \sim b$ means that

$$
|a-b| \leqslant \mathfrak{m}^{N}(U)
$$

By Proposition 9, one can get

$$
\left|\left(J^{\iota} \tilde{\mathcal{G}} \psi_{(\alpha)}, \underline{V} \partial_{z} \psi_{(\alpha)}\right)\right| \leqslant \mathfrak{m}^{N}(U) .
$$

Thus $\left|A_{2}\right| \leqslant \mathfrak{m}^{N}(U)$. 
Estimate of $A_{3}$. It is easy to obtain $\left|A_{3}\right| \leqslant \mathfrak{m}^{N}(U)$ from the definition of $\mathcal{S}_{\alpha}^{2}$ and $\mathcal{E}^{N}(U)$.

Estimate of $A_{4}$. By definition

$$
A_{4}=\left(J^{\iota} \partial_{z}\left(\underline{V} \eta_{(\alpha)}\right),(1+\eta) \mathcal{K}_{(\alpha)} \eta_{\langle\check{\alpha}\rangle}\right)-\left(J^{\iota}\left(\underline{V} \partial_{z}\right) \psi_{(\alpha)}, \tilde{\mathcal{G}}_{(\alpha)} \psi_{\langle\check{\alpha}\rangle}\right)
$$

It is easy to check that

$$
\left|\left(J^{\iota} \partial_{z}\left(\underline{V} \eta_{(\alpha)}\right),(1+\eta) \mathcal{K}_{(\alpha)} \eta_{\langle\check{\alpha}\rangle}\right)\right| \leqslant\left|J^{\iota} \partial_{z}\left(\underline{V} \eta_{(\alpha)}\right)\right|_{2}\left|(1+\eta) \mathcal{K}_{(\alpha)} \eta_{\langle\check{\alpha}\rangle}\right|_{2} \leqslant \mathfrak{m}^{N}(U) .
$$

On the other hand,

$$
\begin{aligned}
& \left|\left(J^{\iota}\left(\underline{V} \partial_{z}\right) \psi_{(\alpha)}, \tilde{\mathcal{G}}_{(\alpha)} \psi_{(\check{\alpha})}\right)\right| \\
= & \left|\left((1+|D|)^{-\frac{1}{2}} \partial_{z} \psi_{(\alpha)},(1+|D|)^{\frac{1}{2}}\left(\underline{V} J^{\iota} \mathcal{G}_{(\alpha)} \psi_{\langle\check{\alpha}\rangle}\right)\right)\right| \leqslant \mathfrak{m}^{N}(U) .
\end{aligned}
$$

Thus, we obtain the estimate for $A_{4}$.

Estimate of $A_{5}$. This can be obtained by combining

$$
\left|\tilde{\mathcal{G}}_{(\alpha)} \psi_{\langle\breve{\alpha}\rangle}\right|_{2} \leqslant \mathfrak{m}^{N}(U) \text { and }\left|(1+\eta) \mathcal{K}_{(\alpha)} \zeta_{\langle\check{\alpha}\rangle}\right|_{2} \leqslant \mathfrak{m}^{N}(U) .
$$

Estimate of $B_{1}$. It is noted that

$$
B_{1}=\left(J^{\iota} R_{\alpha},\left((1+\eta) \mathfrak{a}-\partial_{z}\left(\tilde{\mathcal{K}} \partial_{z} \eta_{(\alpha)}\right)\right)\right)+\left(J^{\iota} S_{\alpha}, \tilde{\mathcal{G}} \psi_{(\alpha)}\right)
$$

From Proposition 13, one can get

$$
\left|\left(J^{\iota} R_{\alpha},\left((1+\eta) \mathfrak{a}-\partial_{z}\left(\tilde{\mathcal{K}} \partial_{z} \eta_{(\alpha)}\right)\right)\right)\right| \leqslant \mathfrak{m}^{N}(U),
$$

and from Proposition 5, one has

$$
\left|\left(J^{l} S_{\alpha}, \tilde{\mathcal{G}} \psi_{(\alpha)}\right)\right| \leqslant\left\|\mathfrak{B} J^{l} S_{\alpha}\right\|_{2}\left\|\mathfrak{B} \tilde{\mathcal{G}} \psi_{(\alpha)}\right\|_{2} \leqslant \mathfrak{m}^{N}(U)
$$

Therefore, $\left|B_{1}\right| \leqslant \mathfrak{m}^{N}(U)$.

Estimate of $B_{2}$. It follows from the definition of $S_{\alpha}^{\prime}$, Proposition 5 and Remark 3 that

$$
\left|B_{2}\right|=\left|\left(J^{\iota} S_{\alpha}^{\prime}, \tilde{\mathcal{G}} \psi_{(\alpha)}\right)\right| \leqslant\left\|\mathfrak{B} J^{\iota} S_{\alpha}^{\prime}\right\|_{2}\left\|\mathfrak{B} \psi_{(\alpha)}\right\|_{2} \leqslant \mathfrak{m}^{N}(U) .
$$

From the above inequalities, for all $1 \leqslant|\alpha| \leqslant N$ we have

$$
\frac{d}{d t}\left(\mathcal{F}^{\alpha}\left(U^{\iota}\right)+\left(U_{(\alpha)}^{\iota}, \mathcal{S}_{\alpha}^{2} U_{\langle\check{\alpha}\rangle}^{\iota}\right)\right) \leqslant \mathfrak{m}^{N}\left(U^{\iota}\right) .
$$

If $\alpha=0$, we rewrite the system as 


$$
\left\{\begin{array}{l}
\eta_{t}-J^{\iota} \mathcal{G}[0] \psi+J^{\iota} \mathcal{N}_{1}(U)=0 \\
\psi_{t}+J^{\iota}\left(1-\partial_{z z}\right) \eta+J^{\iota} \mathcal{N}_{2}(U)=0
\end{array}\right.
$$

where $\mathcal{N}_{1}(U)$ and $\mathcal{N}_{2}(U)$ are given by

$$
\begin{aligned}
\mathcal{N}_{1}(U)= & \mathcal{G}[0] \psi-\mathcal{G}[\eta] \psi, \\
\mathcal{N}_{2}(U)= & \frac{1}{2}\left(|\nabla \psi|^{2}-\frac{\left(\mathcal{G}[\eta] \psi+\eta_{z} \psi_{z}\right)^{2}}{1+\eta_{z}^{2}}\right) \\
& -\left(v\left(\frac{J}{2 \pi(1+\eta)}\right)-v\left(\frac{J}{2 \pi}\right)\right)+\kappa+\left(\partial_{z z}-1\right) \eta .
\end{aligned}
$$

Taking the $L^{2}$ product of (34) with $\left(\left(1-\partial_{z z}\right) \eta, \mathcal{G}[0] \psi\right)$ yields

$$
\frac{d}{d t} \mathcal{F}^{0}(U) \leqslant M[\eta]\left\|\mathcal{N}_{1}(U)\right\|_{H^{1}}\|\eta\|_{H^{1}}+M[\eta]\left\|\mathfrak{B} \mathcal{N}_{2}(U)\right\|_{2}\|\mathfrak{B} \psi\|_{2} \leqslant \mathfrak{m}^{N}(U) .
$$

Hence

$$
\begin{aligned}
\frac{d}{d t}\left(\mathcal{F}^{N-1}\left(U^{\iota}\right)\right) & \leqslant \mathfrak{m}^{N}\left(U^{\iota}\right), \\
\frac{d}{d t}\left(\mathcal{F}^{N}\left(U^{\iota}\right)+\sum_{|\alpha|=N}\left(U_{(\alpha)}^{\iota}, \mathcal{S}_{\alpha}^{2} U_{\langle\check{\alpha}\rangle}^{\iota}\right)\right) & \leqslant \mathfrak{m}^{N}\left(U^{\iota}\right) .
\end{aligned}
$$

Defining

$$
\tilde{\mathcal{F}}^{N}\left(U^{\iota}\right):=\mathcal{F}^{N}\left(U^{\iota}\right)+M \mathcal{F}^{N-1}\left(U^{\iota}\right)+\sum_{|\alpha|=N}\left(U_{(\alpha)}^{\iota}, \mathcal{S}_{\alpha}^{2} U_{\langle\check{\alpha}\rangle}^{\iota}\right),
$$

one then has

$$
\frac{d}{d t} \tilde{\mathcal{F}}^{N}\left(U^{\iota}\right) \leqslant \mathfrak{m}^{N}\left(U^{\iota}\right)
$$

Upon noting that

$$
\sum_{|\alpha|=N}\left|\left(U_{(\alpha)}^{\iota}, \mathcal{S}_{\alpha}^{2} U_{\langle\check{\alpha}\rangle}^{\iota}\right)\right| \leqslant \frac{1}{2} \mathcal{F}^{N}\left(U^{\iota}\right)+M[\eta] \mathcal{F}^{N-1}\left(U^{\iota}\right),
$$

one arrives at

$$
\frac{1}{2} \mathcal{F}^{N}\left(U^{\iota}\right) \leqslant \tilde{\mathcal{F}}^{N}\left(U^{\iota}\right) \leqslant M[\eta] \mathcal{F}^{N}\left(U^{\iota}\right)
$$

Due to Lemmas $14,(35)$ is surely the energy estimate. Finally, by a standard compactness argument (see $[16,17])$, one can obtain Theorem 1 . 


\section{Acknowledgments}

This work was supported by the Key Research Program of Frontier Sciences of CAS (No. QYZDBSSW-SYS015) and the Strategic Priority Research Program of the Chinese Academy of Sciences (No. XDB22040203). Z.W. would also like to acknowledge the support from CAS Center for Excellence in Complex System Mechanics.

\section{References}

[1] D.M. Ambrose, N. Masmoudi, The zero surface tension limit of two-dimensional water waves, Commun. Pure Appl. Math. 58 (2005) 1287-1315.

[2] D.M. Ambrose, N. Masmoudi, The zero surface tension limit of three-dimensional water waves, Indiana Univ. Math. J. 58 (2009) 479-521.

[3] B. Alvarez-Samaniego, D. Lannes, Large time existence for 3D water-waves and asymptotics, Invent. Math. 171 (2008) 485-541.

[4] V.I. Arkhipenko, Y.D. Barkov, Experimental study of the breakdown of the cylindrical layer of a magnetizable fluid under the action of magnetic forces, J. Appl. Mech. Tech. Phys. 21 (1980) 98-105.

[5] V.G. Bashtovoi, R. Foigel, Solitary and cnoidal waves in ferrofluid, Magnetohydrodynamics 19 (1983) $160-165$.

[6] V.G. Bashtovoi, A. Rex, R. Foigel, Some nonlinear wave processes in a magnetic fluid, J. Magn. Magn. Mater. 39 (1983) 115-118.

[7] K. Beyer, M. Günther, On the Cauchy problem for a capillary drop. I. Irrotational motion, Math. Methods Appl. Sci. 21 (1998) 1149-1183.

[8] M.G. Blyth, E.I. Părău, Solitary waves on a ferrofluid jet, J. Fluid Mech. 750 (2014) 401-420.

[9] E. Bourdin, J.-C. Bacri, E. Falcon, Observation of axisymmetric solitary waves on the surface of a ferrofluid, Phys. Rev. Lett. 104 (2010) 094502.

[10] D. Christodoulou, H. Lindblad, On the motion of the free surface of a liquid, Commun. Pure Appl. Math. 53 (2000) $1536-1602$.

[11] D. Coutand, S. Shkoller, Well-posedness of the free-surface incompressible Euler equations with or without surface tension, J. Am. Math. Soc. 20 (2007) 829-930.

[12] W. Craig, C. Sulem, Numerical simulation of gravity waves, J. Comp. Physiol. 108 (1993) 73-83.

[13] M.D. Groves, D.V. Nilsson, Spatial dynamics methods for solitary waves on a ferrofluid jet, J. Math. Fluid Mech. (2018) 1-32.

[14] P. Guyenne, E.I. Părău, An operator expansion method for computing nonlinear surface waves on a ferrofluid jet, J. Comp. Physiol. 321 (2016) 414-434.

[15] D. Lannes, Well-posedness of the water-waves equations, J. Am. Math. Soc. 18 (2005) 605-654 (electronic).

[16] D. Lannes, The Water Waves Problem: Mathematical Analysis and Asymptotics, vol. 188, American Mathematical Society, 2013

[17] D. Lannes, A stability criterion for two-fluid interfaces and applications, Arch. Ration. Mech. Anal. 208 (2013) $481-567$

[18] H. Lindblad, Well-posedness for the motion of an incompressible liquid with free surface boundary, Ann. Math. 162 (2005) 109-194.

[19] V.I. Nalimov, The Cauchy-Poisson problem, Din. Sploš. Sredy 254 (1974) 104-210.

[20] J. Philip, G. Prakash, T. Jayakumar, P. Kalyanasundaram, B. Raj, Effect of polymer surfactant association on colloidal force, Phys. Rev. E 66 (2002) 011406.

[21] K. Raj, B. Moskowitz, R. Casciari, Advances in ferrofluid technology, J. Magn. Magn. Mater. 143 (1995) 174-180.

[22] D. Rannacher, A. Engel, Cylindrical Korteweg-de Vries solitons on a ferrofluid surface, New J. Phys. 8 (2006) $108-123$.

[23] C. Scherer, A.M. Figueiredo Neto, Ferrofluids: properties and applications, Braz. J. Phys. 35 (2005) $718-727$.

[24] J. Shatah, C. Zeng, Geometry and a priori estimates for free boundary problems of the Euler equation, Commun. Pure Appl. Math. 61 (2008) 698-744.

[25] S. Wu, Well-posedness in Sobolev spaces of the full water wave problem in 2-D, Invent. Math. 130 (1997) 39-72.

[26] S. Wu, Well-posedness in Sobolev spaces of the full water wave problem in 3-D, J. Am. Math. Soc. 12 (1999) 445-495.

[27] V.E. Zakharov, Stability of periodic waves of finite amplitude on the surface of a deep fluid, J. Appl. Mech. Tech. Phys. 2 (1968) 190-194. 